\title{
Recent Advances in ARCH Modelling
}

\author{
Liudas Giraitis $^{1 \star}$, Remigijus Leipus ${ }^{2}$, and Donatas Surgailis ${ }^{3}$ \\ 1 University of York, Department of Mathematics and Economics. 1g8@york.ac .uk \\ 2 Vilnius University, Institute of Mathematics and Informatics. \\ Remigijus.Leipus@maf .vu.lt \\ 3 Vilnius Institute of Mathematics and Informatics. sdonatas@ktl.mii.lt
}

\section{Introduction}

Econometric modelling of financial data received a broad interest in the last 20 years and the literature on $\mathrm{ARCH}$ and related models is vast. Starting with the path breaking works by Engle (1982) and Bollerslev (1986), one of the most popular models became the Generalized AutoRegressive Conditionally Heteroscedastic $(\mathrm{GARCH})$ process. The classical $\operatorname{GARCH}(p, q)$ model is given by equations

$$
r_{t}=\sigma_{t} \varepsilon_{t}, \quad \sigma_{t}^{2}=\alpha_{0}+\sum_{i=1}^{p} \beta_{i} \sigma_{t-i}^{2}+\sum_{j=1}^{q} \alpha_{j} r_{t-j}^{2}
$$

where $\alpha_{0}>0, \alpha_{j} \geq 0, \beta_{i} \geq 0, p \geq 0, q \geq 0$ are model parameters and $\left\{\varepsilon_{j}, j \in \mathbf{Z}\right\}$ are independent identically distributed (i.i.d.) zero mean random variables. The variables $r_{t}, \sigma_{t}, \varepsilon_{t}$ in (65) are usually interpreted as financial $(\log )$ returns $\left(r_{t}\right)$, their volatilities or conditional standard deviations $\left(\sigma_{t}\right)$, and so-called innovations or shocks $\left(\varepsilon_{t}\right)$, respectively; in (65) the innovations are supposed to follow a certain fixed distribution (e.g., standard normal). Later, a number of modifications of (65) were proposed, which account for asymmetry, leverage effect, heavy tails and other "stylized facts". For statistical and econometric aspects of ARCH modelling, see the surveys of Bollerslev et al. (1992), Shephard (1996), Bera and Higgins (1993), Bollerslev et al. (1994); for specific features of modelling the financial data, including ARCH, see Pagan (1996), Rydberg (2000), Mikosch (2003). Berkes et al. (2002b) review some recent results. One should mention here, besides the classical reference to Taylor (1986), the related monographs by Gouriéroux (1997), Fan and Yao (2002), Tsay (2002). Let us note that the GARCH model for returns is also related to the Autoregressive Conditional Duration (ACD) model proposed by Engle and Russell (1998) for modelling of durations between events.

\footnotetext{
* Supported by the ESRC grant R000239538.
} 
Under some additional conditions, similarly as in the case of ARMA models, the GARCH model can be written as $\operatorname{ARCH}(\infty)$ model (see (3) below), i.e., $\sigma_{t}^{2}$ can be represented as a moving average of the past squared returns $r_{s}^{2}, s<t$, with exponentially decaying coefficients (see Bollerslev, 1988) and absolutely summable exponentially decaying autocovariance function.

However, empirical studies of financial data show that sample autocorrelations of power series and volatilities (such as absolute values $\left|r_{t}\right|$ or squares $r_{t}^{2}$ ) remain non-zero for very large lags; see, e.g., Dacorogna et al. (1993), Ding et al. (1993), Baillie et al. (1996a), Ding and Granger (1996), Breidt et al. (1998), Mikosch and Stărică (2003), Andersen et al. (2001). These studies provide a clearcut evidence in favor of models with autocovariances decaying slowly with the lag as $k^{-\gamma}$, for some $0<\gamma<1$.

A number of such models (FIGARCH, LM-ARCH, FIEGARCH) were suggested in the ARCH literature. The long memory property was rigorously established for some of these models including the Gaussian subordinated stochastic volatility model (Robinson, 2001), with general form of nonlinearity, the FIEGARCH and related exponential volatility models (Harvey, 1998; Surgailis and Viano, 2002), the LARCH model (Giraitis et al., 2000c), the stochastic volatility model of Robinson and Zaffaroni (1997, 1998). The long memory property (and even the existence of stationary regime) of some other models (FIGARCH, LM-ARCH) has not been theoretically established; see Giraitis et al. (2000a) Mikosch and Stărică (2000, 2003), Kazakevičius et al. (2004). Covariance long memory was also proved for some regime switching SV models (Liu, 2000; Leipus et al., 2005). One should also mention that some authors (Mikosch and Stărică, 1999, 2004) argue that the observed long memory in sample autocorrelations can be explained by short memory GARCH models with structural breaks and/or slowly changing trends.

The present paper reviews some recent theoretical findings on $\mathrm{ARCH}$ type models. We focus mainly on covariance stationary models which display empirically observed properties known as "stylized facts". One of the major issues to determine is whether the corresponding model $r_{t}^{2}$ for squares has long memory or short memory, i.e. whether $\sum_{k=0}^{\infty}\left|\operatorname{Cov}\left(r_{k}^{2}, r_{0}^{2}\right)\right|=\infty$ or $\sum_{k=0}^{\infty}\left|\operatorname{Cov}\left(r_{k}^{2}, r_{0}^{2}\right)\right|<\infty$ holds. It is pointed out that for several ARCH-type models the behavior of $\operatorname{Cov}\left(r_{k}^{2}, r_{0}^{2}\right)$ alone is sufficient to derive the limit distribution of $\sum_{j=1}^{N}\left(r_{j}^{2}-E r_{j}^{2}\right)$ and statistical inferences, without imposing any additional (e.g. mixing) assumptions on the dependence structure.

The review discusses $\mathrm{ARCH}(\infty)$ processes and their modifications such as linear ARCH (LARCH), bilinear models, long memory EGARCH and stochastic volatility, regime switching SV models, random coefficient $\mathrm{ARCH}$ and aggregation. We give an overview of the theoretical results on the existence of a stationary solution, dependence structure, limit behavior of partial sums, leverage effect and long memory property of these models. Statistical estimation of ARCH parameters and testing for change-points are also discussed. 


\section{$2 \operatorname{ARCH}(\infty)$}

A random sequence $\left\{r_{t}, t \in \mathbf{Z}\right\}$ is said to satisfy $\operatorname{ARCH}(\infty)$ equations if there exists a sequence of i.i.d. zero mean random variables $\left\{\varepsilon_{t}, t \in \mathbf{Z}\right\}$ and a deterministic sequence $b_{j} \geq 0, j=0,1, \ldots$ such that for any $t$

$$
r_{t}=\sigma_{t} \varepsilon_{t}, \sigma_{t}^{2}=b_{0}+\sum_{j=1}^{\infty} b_{j} r_{t-j}^{2} .
$$

Clearly, if $E\left(\varepsilon_{t} \mid r_{s}, s<t\right)=0, E\left(\varepsilon_{t}^{2} \mid r_{s}, s<t\right)=1$ then $r_{t}$ has conditional mean zero and a random conditional variance $\sigma_{t}^{2}$, i.e.

$$
E\left(r_{t} \mid r_{s}, s<t\right)=0, \quad \operatorname{Var}\left(r_{t} \mid r_{s}, s<t\right)=\sigma_{t}^{2} .
$$

The general framework leading to the model (2) was introduced by Robinson (1991) in the context of testing for strong serial correlation and has been subsequently studied by Kokoszka and Leipus (2000) in the change-point problem context. The class of $\mathrm{ARCH}(\infty)$ models include the finite order $\mathrm{ARCH}$ and GARCH models of Engle (1982) and Bollerslev (1986). For instance, the $\operatorname{GARCH}(p, q)$ process $\left\{r_{t}, t \in \mathbf{Z}\right\}$ of (65) can be written as $r_{t}=\sigma_{t} \varepsilon_{t}$,

$$
\sigma_{t}^{2}=(1-\beta(1))^{-1} \alpha_{0}+(1-\beta(L))^{-1} \alpha(L) r_{t}^{2},
$$

where $\beta(L)=\beta_{1} L+\ldots+\beta_{p} L^{p}$ and $L$ stands for the back-shift operator, $L^{j} X_{t}=$ $X_{t-j}$. This leads to $\operatorname{ARCH}(\infty)$ representation (2) for $\operatorname{GARCH}(p, q)$ model with $b_{0}=(1-\beta(1))^{-1} \alpha_{0}$ and with positive exponentially decaying weights $b_{j}, j \geq 1$ defined by the generating function $\alpha(z) /(1-\beta(z))=\sum_{i=1}^{\infty} b_{i} z^{i}$. It is interesting to note that the non-negativity of the regression coefficients $\alpha_{j}, \beta_{j}$ in (65) is not necessary for non-negativity of $b_{j}$ in the corresponding $\operatorname{ARCH}(\infty)$ representation, see Nelson and Cao (1992).

\subsection{Existence of Second and Fourth Order Stationary Solutions}

One of the first questions which usually arise in the study of recursion equations of the type (2) is to find conditions for the existence of a stationary solution. We first discuss conditions on the coefficients $b_{j}$ and the random variables $\varepsilon_{t}$ which guarantee the existence of a stationary solution to equations (2) with finite second or fourth moments.

Formally, recursion relations (2) give the following Volterra series expansion of $r_{t}^{2}$ :

$$
\begin{aligned}
r_{t}^{2} \equiv \varepsilon_{t}^{2} \sigma_{t}^{2} & =\varepsilon_{t}^{2} b_{0}\left(1+\sum_{k=1}^{\infty} \sum_{j_{1}, \ldots, j_{k}=1}^{\infty} b_{j_{1}} \ldots b_{j_{k}} \varepsilon_{t-j_{1}}^{2} \ldots \varepsilon_{t-j_{1}-\cdots-j_{k}}^{2}\right) \\
& =\varepsilon_{t}^{2} b_{0}\left(1+\sum_{k=1}^{\infty} \sum_{-\infty<s_{k}<\cdots<s_{1}<t}^{\infty} b_{t-s_{1}} b_{s_{1}-s_{2}} \ldots b_{s_{k-1}-s_{k}} \varepsilon_{s_{1}}^{2} \ldots \varepsilon_{s_{k}}^{2}\right) .
\end{aligned}
$$


By taking the expectation on both sides and using the independence of $\varepsilon_{t}$ 's, one obtains

$$
\begin{aligned}
E r_{t}^{2} & =\left(E \varepsilon_{t}^{2}\right) b_{0}\left\{1+\sum_{k=1}^{\infty} \sum_{-\infty<s_{k}<\cdots<s_{1}<t}^{\infty} b_{t-s_{1}} b_{s_{1}-s_{2}} \ldots b_{s_{k-1}-s_{k}} E \varepsilon_{s_{1}}^{2} \ldots E \varepsilon_{s_{k}}^{2}\right\} \\
& =\left(E \varepsilon_{t}^{2}\right) b_{0}\left\{1+\sum_{k=1}^{\infty}\left(E \varepsilon_{0}^{2} \sum_{j=1}^{\infty} b_{j}\right)^{k}\right\}=\frac{b_{0} E \varepsilon_{0}^{2}}{1-E \varepsilon_{0}^{2} \sum_{j=1}^{\infty} b_{j}} .
\end{aligned}
$$

Hence it easily follows that

$$
E \varepsilon_{0}^{2} \sum_{j=1}^{\infty} b_{j}<1
$$

is sufficient for the existence of stationary solution (4) with $E r_{t}^{2}<\infty$. The uniqueness and the necessity of (5) for the existence of such a solution also follow easily, see Kokoszka and Leipus (2000), Giraitis et al. (2000a).

It is also easy to obtain a sufficient condition for the existence of a stationary solution with finite fourth moment. To that end, apply to (4) the norm (Minkowski) inequality: $\left(E\left(\sum_{i} \xi_{i}\right)^{2}\right)^{1 / 2} \leq \sum_{i}\left(E \xi_{i}^{2}\right)^{1 / 2}$. Similarly as above, this yields

$$
\begin{array}{r}
\left.\left(E r_{t}^{4}\right)^{1 / 2} \leq\left(E \varepsilon_{t}^{4}\right)^{1 / 2} \sum_{k=1}^{\infty} \sum_{-\infty<s_{k}<\cdots<s_{1}<t}^{\infty} b_{t-s_{1}} b_{s_{1}-s_{2}} \ldots b_{s_{k-1}-s_{k}}\left(E \varepsilon_{s_{1}}^{4}\right)^{1 / 2} \ldots\left(E \varepsilon_{s_{k}}^{4}\right)^{1 / 2}\right\} \\
=\frac{b_{0}\left(E \varepsilon_{0}^{4}\right)^{1 / 2}}{1-\left(E \varepsilon_{0}^{4}\right)^{1 / 2} \sum_{j=1}^{\infty} b_{j}}
\end{array}
$$

Hence if condition

$$
\left(E \varepsilon_{0}^{4}\right)^{1 / 2} \sum_{j=1}^{\infty} b_{j}<1
$$

is satisfied, then $r_{t}$ of (4) is a fourth order stationary solution to (2), see Giraitis et al. (2000a). A similar norm inequality works in the case of $E\left(r_{t}^{2}\right)^{p}$ and arbitrary $p \geq 1$, yielding a sufficient condition $\left(E\left|\varepsilon_{0}\right|^{2 p}\right)^{1 / p} \sum_{j=1}^{\infty} b_{j}<1$.

Condition (6) is not necessary for the existence of fourth order stationary solution. For example, in the case of $\operatorname{GARCH}(1,1) r_{t}=\varepsilon_{t} \sigma_{t}, \sigma_{t}^{2}=\alpha_{0}+$ $\alpha r_{t-1}^{2}+\beta \sigma_{t-1}^{2}$, (6) translates to $\alpha \lambda_{2}^{1 / 2}+\beta<1, \lambda_{i}=E \varepsilon^{2 i}, i=1,2$, while a fourth order stationary solution is known to exist under the weaker conditions

$$
\alpha \lambda_{1}+\beta<1, \quad \alpha^{2} \lambda_{2}+\beta^{2}<1,
$$

see Karanasos (1999), He and Teräsvirta (1999). To obtain a sufficient and necessary condition in the general case, one needs to study orthogonal Volterra representation of $r_{t}^{2}$. 
The orthogonal Volterra representation (9) of $r_{t}^{2}$ is obtained by centering the innovations in the (nonorthogonal) representation (4), i.e. by replacing the $\varepsilon_{j}^{2}$ 's by $\kappa \zeta_{j}+\lambda_{1}=\varepsilon_{j}^{2}$, where the standardized $\zeta_{j}=\left(\varepsilon_{j}^{2}-E \varepsilon_{j}^{2}\right) / \kappa, \kappa^{2}=\operatorname{Var}\left(\varepsilon_{0}^{2}\right)$ have zero mean and unit variance.

The resulting expression appears rather complicated, but nevertheless it can be identified and studied (Giraitis and Surgailis, 2002). In order to describe it, denote $g_{j}$ the coefficients of the generating function

$$
\sum_{j=0}^{\infty} g_{j} z^{j}=\left(1-\lambda_{1} \sum_{i=1}^{\infty} b_{i} z^{i}\right)^{-1}
$$

More explicitly,

$$
g_{j}=\sum_{k=1}^{j} \lambda_{1}^{k} \sum_{0<i_{1}<\ldots<i_{k-1}<j} b_{i_{1}} b_{i_{2}-i_{1}} \ldots b_{i_{k-2}-i_{k-1}} b_{j-i_{k-1}} \quad(j \geq 1), \quad g_{0}=1 .
$$

Also introduce $h_{j}=\left(\kappa / \lambda_{1}\right) g_{j}, j \geq 1$,

$$
B=\sum_{j=1}^{\infty} b_{j}, \quad H^{2}=\sum_{j=1}^{\infty} h_{j}^{2} .
$$

Then

$$
r_{t}^{2}=\mu+\left(\kappa / \lambda_{1}\right) \mu \sum_{k=1}^{\infty} \sum_{s_{k}<\cdots<s_{2}<s_{1} \leq t} g_{t-s_{1}} h_{s_{1}-s_{2}} \ldots h_{s_{k-1}-s_{k}} \zeta_{s_{1}} \ldots \zeta_{s_{k}},
$$

where $\mu=E r_{t}^{2}=\lambda_{1} b_{0} /\left(1-\lambda_{1} B\right)$. The series (9) converges in mean square if and only if

$$
\lambda_{1} B<1, \quad H<1
$$

hold, and define a stationary solution of (2).

In fact, conditions (10) are sufficient and necessary for the existence of fourth order stationary solution of (2) (Giraitis and Surgailis, 2002). By orthogonality, it easily follows that

$$
\begin{aligned}
\operatorname{Cov}\left(r_{t}^{2}, r_{0}^{2}\right) & =\left(\kappa / \lambda_{1}\right)^{2} \mu^{2} \sum_{k=1}^{\infty} \sum_{s_{k}<\cdots<s_{1} \leq 0} g_{-s_{1}} g_{t-s_{1}} h_{s_{1}-s_{2}}^{2} \ldots h_{s_{k-1}-s_{k}}^{2} \\
& =\left(\kappa / \lambda_{1}\right)^{2} \mu^{2} \sum_{s \leq 0} g_{s} g_{t-s} \sum_{k=1}^{\infty} H^{2(k-1)} \\
& =\frac{\left(\kappa / \lambda_{1}\right)^{2} \mu^{2}}{1-H^{2}} \sum_{s=0}^{\infty} g_{s} g_{s+t} .
\end{aligned}
$$

For the $\operatorname{GARCH}(1,1)$ model 


$$
r_{t}=\varepsilon_{t} \sigma_{t}, \quad \sigma_{t}^{2}=\alpha_{0}+\alpha r_{t-1}^{2}+\beta \sigma_{t-1}^{2}
$$

the above formulas are more explicit. The model itself can be rewritten in the $\operatorname{ARCH}(\infty)$ form

$$
\sigma_{t}^{2}=\alpha_{0}(1-\beta)^{-1}+\alpha \sum_{j=1}^{\infty} \beta^{j-1} r_{t-j}^{2} .
$$

In this case, $g_{j}=\alpha \lambda_{1}\left(\lambda_{1} \alpha+\beta\right)^{j-1}$, conditions (7) and (10) coincide, and (9) becomes

$$
\begin{aligned}
r_{t}^{2}=\mu & +\mu \kappa \lambda_{1}^{-1} \zeta_{t}\left(1+\sum_{k=1}^{\infty}(\alpha \kappa / \gamma)^{k} \sum_{s_{k}<\cdots<s_{1}<t} \gamma^{t-s_{k}} \zeta_{s_{1}} \ldots \zeta_{s_{k}}\right) \\
& +\mu \sum_{k=1}^{\infty}(\alpha \kappa / \gamma)^{k} \sum_{s_{k}<\cdots<s_{1}<t} \gamma^{t-s_{k}} \zeta_{s_{1}} \ldots \zeta_{s_{k}},
\end{aligned}
$$

where $\gamma=\lambda_{1} \alpha+\beta, \mu=\alpha_{0} \lambda_{1} /(1-\gamma)$. From (12) or (11) one can explicitly find the variance and covariance function of the $\operatorname{GARCH}(1,1)$ model in terms of the coefficients $\alpha_{0}, \alpha, \beta$ and the moments $\lambda_{1}, \lambda_{2}$ :

$$
\begin{aligned}
\operatorname{Var} r_{0}^{2} & =\frac{\alpha_{0}^{2} \kappa^{2}\left(1-\gamma^{2}+\gamma \alpha \lambda_{1}\right)}{(1-\gamma)^{2}\left(1-\gamma^{2}-\alpha^{2} \kappa^{2}\right)} \\
\operatorname{Cov}\left(r_{k}^{2}, r_{0}^{2}\right) & =\frac{\alpha_{0}^{2} \alpha \lambda_{1} \kappa^{2}\left(1-\gamma^{2}+\gamma \alpha \lambda_{1}\right)}{(1-\gamma)^{2}\left(1-\gamma^{2}-\alpha^{2} \kappa^{2}\right)} \gamma^{k-1}, \quad k \geq 1
\end{aligned}
$$

which were also obtained in Teräsvirta (1996). We also note an alternative approach in Kazakevičius et al. (2004) to the problem of the existence of fourth order stationary solution of $\operatorname{ARCH}(\infty)$, which leads to equivalent necessary and sufficient conditions as (10).

For necessary and sufficient conditions of the existence of high order moments for the family of GARCH processes see Ling and McAleer (2002a, 2002b). Ling and McAleer (2003a) studied theoretical properties of the multivariate ARMA-GARCH model.

\subsection{Dependence Structure, Association and Limit Theorems}

The equation (11) for the covariance of $\mathrm{ARCH}(\infty)$ squares $r_{t}^{2}$ allows to directly study its summability and decay properties. From (8) and the summability of $b_{j}$ 's it follows the summability of $g_{j}$ 's which in turn implies by (11) the summability of the autocovariances of $r_{t}^{2}$ :

$$
\sum_{k=-\infty}^{\infty} \operatorname{Cov}\left(r_{k}^{2}, r_{0}^{2}\right)<\infty
$$

(Note that $\operatorname{Cov}\left(r_{k}^{2}, r_{0}^{2}\right) \geq 0$ for all $k$, which follows from (11) and also from the associativity property of $r_{t}^{2}$, see below.) Equation (13) indicates that the 
squares $r_{t}^{2}$ of a fourth order stationary solution of $A R C H(\infty)$ have short memory. The above mentioned papers Giraitis et al. (2000a), Giraitis and Surgailis (2002) also prove that a hyperbolic decay $b_{j} \sim C j^{-\gamma}$ with $\gamma>1$ implies

$$
\operatorname{Cov}\left(r_{k}^{2}, r_{0}^{2}\right) \asymp k^{-\gamma} .
$$

(Here and below, $x_{k} \sim y_{k}$ means $x_{k} / y_{k} \rightarrow 1$ while $x_{k} \asymp y_{k}$ means that there are positive constants $C_{1}$ and $C_{2}$ such that $C_{1} y_{k}<x_{k}<C_{2} y_{k}$ for all $k$.) Thus, even though condition (6) implies absolute summability of the covariances, it allows for a very slow rate of decay of the autocorrelation function when $\gamma>1$ is close to 1 . The last property may be characterized as moderate memory. Near epoch dependence and moderate memory property of the so-called HYGARCH model were studied by Davidson (2004).

The above discussion basically concerns second-order properties of $r_{t}^{2}$ only. Some further insight about these properties can be obtained from the moving average representation

$$
r_{t}^{2}=E r_{t}^{2}+\sum_{j=0}^{\infty} g_{j} \nu_{t-j}
$$

where $g_{j}(8)$ and $\nu_{t} \equiv \sigma_{t}^{2}\left(\varepsilon_{t}^{2}-E \varepsilon_{t}^{2}\right)$ are martingale differences. The above representation is a direct consequence of (9), from which the $\nu_{t}$ 's can be also expressed as a Volterra series in the standardized variables $\zeta_{s}, s<t$. Of course, (14) yields the same covariance formula as (11). On the other hand, the $\nu_{t}$ 's are not independent, meaning that "higher order" dependence and distributional properties of (14) may be very different from the usual moving average in i.i.d. random variables.

$\operatorname{ARCH}(\infty)$ sequences have important property of associativity. A random sequence $\left\{X_{t}\right\}$ is said to be associated (or positively correlated) if the inequality

$$
\operatorname{Cov}\left(f\left(X_{t_{1}}, \ldots, X_{t_{n}}\right), g\left(X_{t_{1}}, \ldots, X_{t_{n}}\right)\right) \geq 0
$$

holds for any coordinate nondecreasing functions $f, g: \mathbf{R}^{n} \rightarrow \mathbf{R}$ and any $t_{1}, \ldots, t_{n}, n=1,2, \ldots$ In particular, the covariance function (if it exists) of associated sequence is nonnegative: $\operatorname{Cov}\left(X_{s}, X_{t}\right) \geq 0$ for any $s, t$. Associated sequences are widely encountered in applications, see e.g. Barlow and Proschan (1981), Newman (1984), Cox and Grimmett (1984). Association is a very strong property, under which uncorrelatedness implies independence similarly as in Gaussian case. A number of limit theorems have been proved for associated sequences under covariance restrictions only. One of the most celebrated results, due to Newman and Wright (1981), says that if $\left\{X_{t}, t \in \mathbf{Z}\right\}$ is strictly stationary and associated and $\sigma^{2}=\sum_{t \in \mathbf{Z}} \operatorname{Cov}\left(X_{0}, X_{t}\right)<\infty$ then the partial sums' process

$$
\left\{N^{-1 / 2} \sum_{t=1}^{[N \tau]}\left(X_{t}-E X_{t}\right), \tau \in[0,1]\right\} \rightarrow_{D[0,1]}\{\sigma W(\tau), \tau \in[0,1]\}
$$


in the Skorokhod space $D[0,1]$, where $\{W(\tau)\}$ is a standard Brownian motion.

It is well known that independent random variables are associated, and that this property is preserved by coordinate-nondecreasing (nonlinear) transformations. In particular, the $\operatorname{ARCH}(\infty)$ process of (4) is a coordinatenondecreasing transformation of the i.i.d. sequence $\left\{\varepsilon_{t}^{2}\right\}$. It is clear from nonnegativity $b_{j} \geq 0, j \geq 0$ that $r_{t}^{2}$ can only increase if any of $\varepsilon_{s}^{2}, s \leq t$ on the r.h.s. of (4) is replaced by some larger quantity. Therefore the $A R C H(\infty)$ process (4) is associated.

An immediate consequence of (15), (13) and the association property of is the functional central limit theorem for squares $r_{t}^{2}$ of $\operatorname{ARCH}(\infty)$ :

$$
\left\{N^{-1 / 2} \sum_{t=1}^{[N \tau]}\left(r_{t}^{2}-E r_{t}^{2}\right), \tau \in[0,1]\right\} \rightarrow_{D[0,1]}\{\sigma W(\tau), \tau \in[0,1]\}
$$

where $\sigma^{2}$ equals to the sum in (13). This result is quite surprising given a rather complicated nonlinear structure of $\operatorname{ARCH}(\infty)$, since it holds for any stationary solution $r_{t}$ such that $E r_{t}^{4}<\infty$. Giraitis et al. (2000a) obtained a similar result by using finite memory approximation to $\operatorname{ARCH}(\infty)$.

It seems that the implications of association property to the study of ARCH models have been not yet fully explored. This remark applies e.g. to the covariance structure and dependence properties of general nonlinear transformations of $\mathrm{ARCH}(\infty)$, Rosenthal inequalities, rate of convergence, empirical processes and many other questions. See the dissertation of Louichi (1998) for references.

\subsection{Stationary Solution of $\operatorname{ARCH}(\infty)$ Without Moment Assumptions}

A rather unusual feature of $\mathrm{ARCH}$ equations is the fact that they may admit a stationary solution which does not have any moments, even if the i.i.d. "shocks" $\varepsilon_{t}$ 's are $N(0,1)$. In such case, the Volterra series (4) converge in probability but not in any moment sense, and the properties of the infinite series are much more difficult to study. Nelson (1990) showed, using the theory of products of random matrices, that a necessary and sufficient condition for the existence of a strictly stationary $\operatorname{GARCH}(1,1)$ process is

$$
E \log \left(\alpha \varepsilon_{0}^{2}+\beta\right)<0 .
$$

This condition is of course much weaker than any of conditions (5), (6), (7) given above (which imply in particular the existence of finite moment $E r_{t}^{2}<$ $\infty$ or $\left.\operatorname{Er}_{t}^{4}<\infty\right)$. Nelson's result was extended to the $\operatorname{GARCH}(p, q)$ case by Bougerol and Picard (1992), who showed that, under condition $E \varepsilon_{0}^{2}=1$, a stationary solution to (65) exists if and only if the top Lyapunov exponent $\gamma$ is strictly negative. Moreover, in such case there exists only one stationary $\operatorname{GARCH}(p, q)$ process. The top Lyapunov exponent is defined by 


$$
\gamma=\lim _{n \rightarrow \infty} n^{-1} \log \left\|A_{1} \cdots A_{n}\right\|,
$$

where $\left\{A_{k}\right\}$ are i.i.d. $(p+q-1) \times(p+q-1)$ random matrices (depending only on $\left.\varepsilon_{k}^{2}\right)$ such that the $(p+q-1)$-valued process $X_{t}=\left(\sigma_{t+1}^{2}, \ldots, \sigma_{t-p+2}^{2}, r_{t}^{2}, \ldots\right.$, $\left.r_{t-q+2}^{2}\right)^{\prime}$ satisfies the random coefficient matrix $\operatorname{AR}(1)$ equation

$$
X_{t}=A_{t} X_{t-1}+B,
$$

with $B=\left(\alpha_{0}, 0, \ldots, 0\right)^{\prime}$; see Bougerol and Picard (1992).

Further progress in this direction was made by Kazakevičius and Leipus (2002), who discussed the general case of $\operatorname{ARCH}(\infty)$. They observed that the volatility can be written as

$$
\sigma_{t}^{2}=b_{0}\left(1+\sum_{n=1}^{\infty} \sigma_{t, n}^{2}\right)
$$

the convergence of the series being equivalent to the existence of a stationary solution $r_{t}=\varepsilon_{t} \sigma_{t}$, where

$$
\sigma_{t, n}^{2}=\sum_{k=1}^{n} \sum_{\substack{i_{1}, \ldots, i_{k} \geq 1 \\ i_{1}+\cdots+i_{k}=n}} b_{i_{1}} b_{i_{2}} \cdots b_{i_{k}} \varepsilon_{t-i_{1}}^{2} \varepsilon_{t-i_{1}-i_{2}}^{2} \cdots \varepsilon_{t-i_{1}-\cdots-i_{k}}^{2} \quad(n \geq 1),
$$

$\sigma_{t, n}^{2}=0(n \leq 0)$, satisfy the recurrent equation

$$
\sigma_{t, n}^{2}=\varepsilon_{t-n}^{2} \sum_{i=1}^{n} b_{i} \sigma_{t, n-i}^{2}, \quad n \geq 1
$$

Equation (20) is a "stochastic" version of the corresponding equation satisfied by $g_{n}=E \sigma_{t, n}^{2}$ of (8) in the case $\lambda_{1}=E \varepsilon_{0}^{2}<\infty$. For a fixed $t$ (say, $t=0$ ), equation (20) can be written in the matrix form:

$$
\Sigma_{n}=B_{n} \Sigma_{n-1},
$$

where $\Sigma_{n}=\left(\sigma_{0, n}^{2}, \sigma_{0, n-1}^{2}, \ldots, \sigma_{0,1}^{2}, 0, \ldots\right)^{\prime}$ and where $\left\{B_{n}\right\}$ are random i.i.d. (infinite) matrices:

$$
B_{n}=\left(\begin{array}{cccc}
b_{1} \varepsilon_{-n}^{2} & b_{2} \varepsilon_{-n}^{2} & b_{3} \varepsilon_{-n}^{2} & \cdots \\
1 & 0 & 0 & \cdots \\
0 & 1 & 0 & \cdots \\
\cdots & \cdots & \cdots & \cdots
\end{array}\right)
$$

Kazakevičius and Leipus (2002) showed that in the $\operatorname{GARCH}(p, q)$ case, the top Lyapunov exponent satisfies

$$
\gamma=-\log R,
$$


where $R=\left(\limsup \sqrt[n]{\sigma_{0, n}^{2}}\right)^{-1} \in[0, \infty]$ is the convergence radius of the random power series

$$
\sigma_{t}^{2}(z)=b_{0}\left(1+\sum_{n=1}^{\infty} \sigma_{t, n}^{2} z^{n}\right),
$$

see equation (19). It turns out that in general $\mathrm{ARCH}(\infty)$ case, $R$ is nonrandom and therefore $\gamma$ defined by (22) can be considered an the analog of of the top Lyapunov exponent. Similarly as in the GARCH case, $\gamma<0$ implies the existence of a stationary solution $r_{t}=\varepsilon_{t} \sigma_{t}$ of $\operatorname{ARCH}(\infty)$, with $\sigma_{t}^{2}$ given by (19). The last condition is of course not necessary and a stationary solution may as well exist if $\gamma=0$. In fact, using the argument of Kazakevičius and Leipus (2003) one can show that a stationary solution of $\mathrm{ARCH}(\infty)$ with finite mean and coefficients $b_{j} \asymp j^{-q}, q>1$ satisfies $\gamma=0$. This shows that vanishing of the top Lyapunov exponent is typical for stationary solutions of $\operatorname{ARCH}(\infty)$ with a power-like decay of coefficients.

Kazakevičius and Leipus (2002) also proved the uniqueness of the above stationary solution under some additional condition on the coefficients $b_{j}$ (which is satisfied, for example, if these coefficients monotonically decay starting with $j$ large enough).

\subsection{Existence of Integrated $\operatorname{ARCH}(\infty)$ Process}

To invoke the widely discussed and notable analogy between ARCH and ARMA, one has to rearrange the $\operatorname{GARCH}(p, q)$ equation $(65)$ so that $r_{t}^{2}$ satisfies $\operatorname{ARMA}(p+q, q)$ equation with martingale difference innovations $\nu_{t}=r_{t}^{2}-\sigma_{t}^{2}$ :

$$
(1-\alpha(L)-\beta(L)) r_{t}^{2}=\alpha_{0}+(1-\beta(L)) \nu_{t}
$$

(we assume $E \varepsilon_{0}^{2}=1$ for simplicity). Then

$$
\sum_{i=1}^{q} \alpha_{i}+\sum_{j=1}^{p} \beta_{j}=1
$$

is the unit root condition. The corresponding GARCH model, called the Integrated $\operatorname{GARCH}(p, q)$, was introduced by Engle and Bollerslev (1986) in analogy with integrated ARMA model in order to explain the observed IGARCH effect in financial data when the estimated parameters $\alpha_{1}, \ldots, \alpha_{q}$ and $\beta_{1}, \ldots, \beta_{p}$ of the (65) model sum up to a value which is close to one. A similar rearrangement of (2) leads to the notion Integrated $A R C H(\infty)$, or $\operatorname{IARCH}(\infty)$, which is defined as a solution to (2) with

$$
E \varepsilon_{0}^{2} \sum_{j=1}^{\infty} b_{j}=1 .
$$

Integrated processes constitute an important class of $\mathrm{ARCH}$ processes where the similarities and the differences between ARCH and ARMA most 
distinctly appear. It is easily seen (see also (5)) that stationarity and (26) imply $E r_{t}^{2}=\infty$ and therefore a stationary $\operatorname{IARCH}(\infty)$ process $r_{t}$ necessarily has infinite variance. Moreover, in this case even the interpretation of $\nu_{t}$ as martingale innovations becomes peculiar as the $\nu_{t}$ 's have infinite unconditional absolute mean. Nevertheless, differently from $\operatorname{AR}(\infty)$ case, the $\operatorname{IGARCH}(\infty)$ equation may admit a stationary solution.

The most famous example of IARCH processes is the FIGARCH process defined by

$$
r_{t}=\sigma_{t} \varepsilon_{t}, \sigma_{t}^{2}=b_{0}+\left(1-(1-L)^{d}\right) r_{t}^{2},
$$

where $b_{0}>0$ and $(1-L)^{d}, 0<d<1$ is the fractional differencing operator. This model, introduced by Baillie et al. (1996a) in order to capture long memory effect in volatility, allows a hyperbolic decay of the coefficients $b_{j}$ which are positive, summable, and satisfy the unit root condition (26). However, the proof of existence of stationary solution to (27) given in Baillie et al. (1996a) does not seem to be correct. The question of the existence a stationary solution to FIGARCH equation (the "FIGARCH problem") is open at the present and seems very hard. See Giraitis et al. (2000a), Mikosch and Stărică (2000, 2003), for discussion and controversies surrounding the FIGARCH case.

Bougerol and Picard (1992) obtained the existence of a stationary Integrated $\operatorname{GARCH}(p, q)$ process as a corollary to their more general result discussed above. Essentially, their conditions (which ensure that the top Lyapunov exponent $\gamma<0$ ) require that all coefficients $\alpha_{i}, \beta_{i}$ are strictly positive. Kazakevičius and Leipus (2003) discussed a similar problem in the general $\operatorname{IARCH}(\infty)$ case, by using the definition of $\gamma(22)$ via the convergence radius $R$. They replaced the positivity condition of the coefficients of Bougerol and Picard (1992) by the requirement that the weights $b_{j}$ decay exponentially, more precisely, that

$$
\sum_{j=1}^{\infty} b_{j} q^{j}<\infty \text { for some } q>1
$$

If $\gamma$ is negative then $r_{t}=\varepsilon_{t} \sigma_{t}$ with $\sigma_{t}(19)$ is a stationary $\operatorname{IARCH}(\infty)$ solution. They also showed (assuming $E \log ^{-} \varepsilon_{0}^{2}<\infty$ ) that if this decay condition of $b_{j}$ is not satisfied (as in the FIGARCH case), then $\gamma=0$. The last result can be considered as a further confirmation of the difficulty of the FIGARCH problem, and indicates the limitations of the random matrices approach to the existence problem of stationary solution of ARCH equations.

Our final remark concerns the question of long memory. The result (13) can be interpreted as the fact that the squares $r_{t}^{2}$ of covariance stationary $\operatorname{ARCH}(\infty)$ always have short memory. However, the last fact does not rule out the possibility that absolute values $\left|r_{t}\right|$ or some (fractional) powers $\left|r_{t}\right|^{\delta}, \delta>0$ may have non-summable autocorrelations, under the same conditions which guarantee the existence of covariance stationary solution and especially in the case of IARCH when $r_{t}^{2}$ has infinite variance. Such possibility seems unlikely, because of the summability of the coefficients $b_{j}$ in $\operatorname{ARCH}(\infty)$ and the associativity property discussed above. Nevertheless, several empirical studies 
indicate that sample autocovariances of absolute powers of asset returns exhibit "maximal memory" for $\delta=1$, see Ding et al. (1993). While absolute powers $\left|r_{t}\right|^{\delta}$ of $\mathrm{ARCH}(\infty)$ are mathematically hard to handle (unless $\delta$ is an even integer), this was one of the reasons for introducing stochastic volatility models, which allow modelling of long memory; see below.

\section{Other ARCH and Related Models}

In a wider sense, the term "ARCH process" refers to the class of processes $r_{t}$ with zero conditional mean $E\left(r_{t} \mid r_{s}, s<t\right)=0$ and the conditional variance $\sigma_{t}^{2}=\operatorname{Var}\left(r_{t} \mid r_{s}, s<t\right)$ being a general function of the past information set $r_{s}, s<t$ (which may also include some additional "exogenous" variables). The possibilities of choices of functional forms of $\sigma_{t}^{2}$ are very numerous, leading to a vast family of $\mathrm{ARCH}$ models and modifications, only part of which is mentioned in our reference list. This review focuses on the probabilistic aspects of $\mathrm{ARCH}$ modelling, and we do not attempt to cover the whole econometric literature on ARCH. Of course, the topics and models discussed below are motivated in some sense by the interests of the authors of the review.

\subsection{The LARCH Model}

The Linear ARCH (LARCH) model, introduced by Robinson (1991), is defined by

$$
r_{t}=\sigma_{t} \varepsilon_{t}, \quad \sigma_{t}=\alpha+\sum_{j=1}^{\infty} \beta_{j} r_{t-j},
$$

where $\left\{\varepsilon_{t}, t \in \mathbf{Z}\right\}$ is an i.i.d. sequence with zero mean and finite variance, and the coefficients $\beta_{j}$ satisfy

$$
\beta_{j} \sim c j^{d-1},
$$

for some $0<d<1 / 2, c>0$. The particular case

$$
r_{t}=\sigma_{t} \varepsilon_{t}, \quad \sigma_{t}=(1-L)^{-d} r_{t}
$$

corresponds to the LARCH equation with $\operatorname{FARIMA}(0, d, 0)$ coefficients.

The main advantage of LARCH is that it allows modelling of long memory as well as some characteristic asymmetries (the "leverage effect"). Both these properties cannot be modeled by the classical $\mathrm{ARCH}(\infty)$ with finite fourth moment. The condition (29) implies only $\sum_{j} \beta_{j}^{2}<\infty$ which is weaker than assumption $\sum_{j} b_{j}<\infty$ for the $\operatorname{ARCH}(\infty)$ model (2). Neither $\alpha$ nor the $\beta_{j}$ are assumed positive and, unlike in $(2), \sigma_{t}$ (not $\sigma_{t}^{2}$ ), is a linear combination of the past values of $r_{t}$, rather than their squares.

A not so pleasant feature of the LARCH model is that $\sigma_{t}$ may be negative or vanish, being a linear combination of martingale differences $r_{t}$ with zero 
mean, and so it lacks some of the usual volatility interpretation. Recently, Koulikov (2003) considered a particular case of LARCH which he claims has the property that $\sigma_{t}>0$ a.s. If Koulikov's claim is correct, this would certainly increase the interest to the LARCH model and statistical inferences.

By the definition (28), the conditional variance is $\operatorname{Var}\left(r_{t} \mid r_{s}, s<t\right)=\sigma_{t}^{2}$, where

$$
\sigma_{t}^{2}=\left(\alpha+\sum_{j=1}^{\infty} \beta_{j} r_{t-j}\right)^{2}
$$

is the squared linear combination of $r_{s}, s<t$ and so the LARCH model formally appears a particular case of Sentana's Quadratic ARCH (QARCH) models (Sentana, 1995). In the QARCH case, the conditional variance is an arbitrary nonnegative second order polynomial of the past information set $r_{s}, s<t$, which may contain both linear and quadratic terms. While the discussion in Sentana (1995) seems limited to finite memory Markov models, his model allows considerable flexibility and asymmetry. It is not clear if the method of Volterra expansions can be applied to the study of the QARCH model, as these expansions might appear intractable.

Long memory properties of the LARCH model were studied in Giraitis et al. (2000c), Giraitis et al. (2004). Similarly as the $\mathrm{ARCH}(\infty)$ case, it is easy to show that a covariance stationary solution $r_{t}$ to (28) exists if and only if

$$
b=\left\{\sum_{j=1}^{\infty} \beta_{j}^{2}\right\}^{1 / 2}<1
$$

(we assume $E \varepsilon^{2}=1$ ), in which case it can be represented by the convergent orthogonal Volterra series

$$
r_{t}=\sigma_{t} \varepsilon_{t}, \quad \sigma_{t}=\alpha\left(1+\sum_{k=1}^{\infty} \sum_{j_{1}, \ldots, j_{k}=1}^{\infty} \beta_{j_{1}} \ldots \beta_{j_{k}} \varepsilon_{t-j_{1}} \ldots \varepsilon_{t-j_{1}-\cdots-j_{k}}\right) .
$$

The above fact holds independently of (29) and requires (30) only. Whence or directly from the LARCH equations (28), the representation

$$
\operatorname{Cov}\left(\sigma_{t}, \sigma_{0}\right)=b^{-2} \sum_{j=1}^{\infty} \beta_{j} \beta_{t+j}
$$

immediately follows, which coincides with the covariance of the linear filter with coefficients $b^{-1} \beta_{j}$. Clearly, under condition (29), $\sigma_{t}$ displays the covariance long memory similar to $\operatorname{FARIMA}(0, d, 0)$ models.

The long memory property of the "observable" process $r_{t}$ is more difficult to establish, as it requires the study of dependence properties of nonlinear functions of $r_{t}$. One of the simplest such functions is $r_{t}^{2}$, however, even in such case, the expression for $\operatorname{Cov}\left(r_{k}^{2}, r_{0}^{2}\right)$ is quite complicated and involves a combinatorial formalism of diagrams (Giraitis et al., 2000c). As shown in this 
paper, under some additional boundedness condition on $b(30)$, the squares $r_{t}^{2}$ of the LARCH model (28), (29) exhibit long memory, in the sense that

$$
\operatorname{Cov}\left(r_{k}^{2}, r_{0}^{2}\right) \sim C k^{2 d-1}
$$

as $k \rightarrow \infty$, where the constant $C>0$ is explicitly written in terms of parameters $\alpha, b, c, d$. The above paper also obtains the convergence of the partial sums' process of $r_{t}^{2}$ to a fractional Brownian motion $W_{d}(\tau), E W_{d}^{2}(\tau)=\tau^{2 d+1}$ :

$$
\left\{\frac{1}{N^{1 / 2+d}} \sum_{j=1}^{[N \tau]}\left(r_{j}^{2}-E r_{j}^{2}\right)\right\} \rightarrow{ }_{D[0,1]} \quad\left\{c_{d} W_{d}(\tau)\right\}
$$

where $c_{d}$ is a positive constant. Similar results under increasingly stringent bounds on $b(30)$ were proved for arbitrary powers $r_{t}^{k}, \sigma_{t}^{k}, k=2,3, \ldots$.

These result indicate that long memory properties of LARCH may be somewhat similar to the properties of linear moving average process with coefficients (29). In fact, the first term of the expansion (31) (corresponding to $k=1$ ) is exactly the linear process $\sum_{j=1}^{\infty} \beta_{j} \varepsilon_{t-j}$, up to the constant $\alpha$. The nonlinearity of the LARCH comes out when analyzing the behavior of higher-order multiple sums in (31). It turns out that every term $\sum_{j_{1}, \ldots, j_{k}} \beta_{j_{1}} \ldots \beta_{j_{k}} \varepsilon_{t-j_{1}} \ldots \varepsilon_{t-j_{k}}$ behaves similarly as the first (linear) term and contributes to the limiting constants $C$ and $c_{d}$ in (32) and (33) respectively, although these "contributions decay geometrically" with $k$. Further important results on long memory LARCH processes can be found in Berkes and Horváth (2003b).

Short memory versions $\operatorname{LARCH}(p), \operatorname{GLARCH}(p, q)$ of $(28)$ can be introduced, similarly as in $\operatorname{ARCH}(\infty)$ case. In fact, $\operatorname{LARCH}(1)$ turns out to be a particular case of the asymmetric ARCH model of Engle (1990), and $\operatorname{GLARCH}(p, q)$ a particular case of Sentana's QARCH model. As mentioned above, the main reasons for introducing these models was the desire to model asymmetric behavior, in particular, the leverage effect. This effect, first described by Black (1976), is the empirically observed property for volatility of asset returns to move in the opposite direction to returns, after a delay, as happens when the conditional variance is negatively correlated with past returns. Engle (1990) and Sentana (1995) discussed the (potential) leverage property in their models. For the LARCH model, the leverage property was rigorously proved in Giraitis et al. (2004). They showed that if the coefficient $\alpha$ and $\beta_{1}, \ldots, \beta_{k}$ have opposite signs, i.e.

$$
\alpha \beta_{j}<0, \quad j=1, \ldots, k,
$$

and some additional conditions are satisfied, then $\sigma_{t}^{2}$ and $r_{t-1}, \ldots, r_{t-k}$ are negatively correlated:

$$
\operatorname{Cov}\left(\sigma_{t}^{2}, r_{t-j}\right)<0, \quad j=1, \ldots, k,
$$


where $k=1,2, \ldots$ can be arbitrary.

A heuristic explanation of this phenomenon from the $\mathrm{LARCH}$ equations is the following. Consider LARCH(1) $\sigma_{t}=\alpha+\beta_{1} r_{t-1}$ and $\alpha>0, \beta_{1}<0$. Clearly an increase of $r_{t-1}$ results in a decrease of $\sigma_{t}$ because of $\beta_{1}<0$. On the other hand, because of $\alpha>0, \sigma_{t}$ spends more time on the positive side, or $\sigma_{t}>0$ is more likely to happen than $\sigma_{t}<0$. This means that a decrease of $\sigma_{t}$ is more likely to cause a decrease of $\sigma_{t}^{2}$, too, or that $r_{t-1}$ and $\sigma_{t}^{2}$ should be negatively correlated.

Giraitis et al. (2003) developed $R / S$-type tests for long memory in LARCH processes against their short memory counterparts. Giraitis et al. (2000b) discussed the estimation of the long memory parameter in the LARCH model.

\subsection{Bilinear Process}

Formally, the classes AR, ARCH, LARCH (at least, their finite memory counterparts ARMA, GARCH, GLARCH) all belong to the general class of bilinear models introduced by Granger and Andersen (1978). The existing literature on bilinear time series models is quite large, see the monographs by Subba Rao and Gabr (1984) and Terdik (1999), however, it does not have much in common with the ARCH literature, probably because it focuses on homoscedastic case. Giraitis and Surgailis (2002) studied the heteroscedastic bilinear equation

$$
X_{t}=\zeta_{t}\left(a_{0}+\sum_{j=1}^{\infty} a_{j} X_{t-j}\right)+c_{0}+\sum_{j=1}^{\infty} c_{j} X_{t-j},
$$

where $\left\{\zeta_{t}, t \in \mathbf{Z}\right\}$ are i.i.d. random variables, with zero mean and variance 1 , and $a_{j}, c_{j}, j \geq 0$ are real (not necessary nonnegative) coefficients. Equation (34) appears naturally when studying the class of processes with the property that the conditional mean $\mu_{t}=E\left(X_{t} \mid X_{s}, s<t\right)$ is a linear combination of $X_{s}, s<t$, and the conditional variance $\sigma_{t}^{2}=\operatorname{Var}\left(X_{t} \mid X_{s}, s<t\right)$ is the square of a linear combinations of $X_{s}, s<t$, as it is in the case of (34):

$$
\sigma_{t}^{2}=\left(a_{0}+\sum_{j=1}^{\infty} a_{j} X_{t-j}\right)^{2}, \quad \mu_{t}=c_{0}+\sum_{j=1}^{\infty} c_{j} X_{t-j} .
$$

Clearly, the case $a_{j} \equiv 0, j \geq 1$ gives the linear $\operatorname{AR}(\infty)$ equation, while $c_{j} \equiv$ $0(j \geq 0)$ results in the LARCH equation $(28)$.

It is less obvious that the $\operatorname{ARCH}(\infty)$ equation (2) is also a special case of the bilinear equation (34). To see this, put $X_{t}=r_{t}^{2}, \zeta_{t}:=\left(\varepsilon_{t}^{2}-\lambda_{1}\right) / \kappa, \lambda_{1}=$ $E \varepsilon_{0}^{2}, \kappa^{2}=\operatorname{Var}\left(\varepsilon_{0}^{2}\right)$. Then (2) can be rewritten as

$$
X_{t}=\zeta_{t}\left(\kappa b_{0}+\kappa \sum_{j=1}^{\infty} b_{j} X_{t-j}\right)+\lambda_{1} b_{0}+\lambda_{1} \sum_{j=1}^{\infty} b_{j} X_{t-j}
$$

which is a particular case of (34). 
Heteroscedastic models with non-zero conditional mean (combinations of the type ARMA-ARCH) have been studied in the literature; see e.g. Baillie et al. (1996b), Ling and Li (1998), Teyssière (2000), Li et al. (2002). The paper Giraitis and Surgailis (2002) attempts a systematic study of bilinear models (34) with long memory. For (34), it defines long memory in conditional mean and long memory in conditional variance, and describes a class of fractional bilinear models which exhibit both types of long memory, with arbitrary (fractional) parameters $0<d_{1}, d_{2}<1 / 2$.

The bilinear model (34) in the cases $c_{0} \neq 0$ and $c_{0}=0$ has different properties. The first case (to which the $\operatorname{ARCH}(\infty)$ reduces) does not essentially allow for covariance stationary long memory in (34). Let $c_{0}=0$ below. Introduce the generating functions

$$
G(z):=(1-C(z))^{-1}=\sum_{j=0}^{\infty} g_{j} z^{j}, \quad H(z):=A(z) G(z)=\sum_{j=1}^{\infty} h_{j} z^{j},
$$

where $A(z):=\sum_{j=1}^{\infty} a_{j} z^{j}, C(z):=\sum_{j=1}^{\infty} c_{j} z^{j}$. Under some natural conditions on coefficients $g_{j}, h_{j}$, the most important of which is

$$
H^{2}=\sum_{j=1}^{\infty} h_{j}^{2}<1
$$

it was proved that equation (34) admits a unique stationary and ergodic solution $X_{t}=\zeta_{t} \sigma_{t}+\mu_{t}$ given by the convergent orthogonal Volterra series

$$
\begin{aligned}
\sigma_{t} & =a_{0}\left(1+\sum_{k=1}^{\infty} \sum_{s_{k}<\cdots<s_{1}<t} h_{t-s_{1}} h_{s_{1}-s_{2}} \ldots h_{s_{k-1}-s_{k}} \zeta_{s_{1}} \ldots \zeta_{s_{k}}\right), \\
\mu_{t} & =a_{0} \sum_{k=1}^{\infty} \sum_{s_{k}<\cdots<s_{1}<t} g_{t-s_{1}} h_{s_{1}-s_{2}} \ldots h_{s_{k-1}-s_{k}} \zeta_{s_{1}} \ldots \zeta_{s_{k}} .
\end{aligned}
$$

Note that here $\sigma_{t} \neq \sqrt{\sigma_{t}^{2}}$, similarly as in the LARCH case, as $\sigma_{t}$ may assume negative values. From (35), (36) it follows that the processes $X_{t}, \mu_{t}, \sigma_{t}$ admit the moving average representations:

$$
\sigma_{t}=a_{0}+\sum_{j=1}^{\infty} h_{j} \nu_{t-j}, \quad \mu_{t}=\sum_{j=1}^{\infty} g_{j} \nu_{t-j}, \quad X_{t}=\sum_{j=0}^{\infty} g_{j} \nu_{t-j}
$$

w.r.t. martingale differences $\nu_{s}=\zeta_{s} \sigma_{s}$, implying in particular

$$
E X_{t}=0, \operatorname{Cov}\left(X_{k}, X_{0}\right)=a_{0}^{2}\left(1-H^{2}\right)^{-1} \sum_{j=0}^{\infty} g_{j} g_{j+k}
$$

If $\left|g_{j}\right|$ and $\left|h_{j}\right|$ are summable, then $X_{t}, \mu_{t}$ and $\sigma_{t}$ have short memory (absolutely summable autocovariances). For example, this holds when 


$$
\left(\sum_{j=1}^{\infty}\left|a_{j}\right|^{2}\right)^{1 / 2}+\sum_{j=1}^{\infty}\left|b_{j}\right|^{2}<1 .
$$

The long memory in conditional mean (respectively, in conditional variance) is defined in terms of the coefficients of the linear filters (37), namely

$$
g_{j} \sim C_{1} j^{d_{1}-1} \quad\left(\text { respectively, } \quad h_{j} \sim C_{2} j^{d_{2}-1}\right),
$$

where $C_{i} \neq 0,0<d_{i}<1 / 2, \quad i=1,2$. Giraitis and Surgailis (2002) presented concrete examples of generating functions of the form $C(z)=$ $1-P_{1}(z)(1-z)^{d_{1}}, A(z)=P_{2}(z)(1-z)^{d_{1}-d_{2}}$, where $P_{i}(z), i=1,2$ satisfy some root conditions, for which the corresponding $G(z), H(z)$ satisfy (38). Consequently, the bilinear equation (34) may exhibit double long memory (i.e. long memory both in conditional mean and in conditional variance). A natural question arises which of these two long memories plays a dominating role. The asymptotic behavior of the covariance of $X_{t}$ depends on $d_{1}$ only $\left(\operatorname{Cov}\left(X_{k}, X_{0}\right)\right.$ decays as $\left.k^{2 d_{1}-1}\right)$, so the above question concerns nonlinear functionals, in particular the squared process $X_{t}^{2}$. The answer to this question depends on which of the two quantities $2\left(1-2 d_{1}\right), 1-2 d_{2}$ is larger: if $2\left(1-2 d_{1}\right)<1-2 d_{2}$, then the long memory in conditional mean dominates, meaning that the covariance $\operatorname{Cov}\left(X_{k}^{2}, X_{0}^{2}\right)$ decays as $k^{2\left(2 d_{1}-1\right)}$ and that a suitably normalized sum $\sum_{t=1}^{N}\left(X_{t}^{2}-E X_{t}^{2}\right)$ converges to a non Gaussian distribution, similarly as if $X_{t}$ were a linear FARIMA process. On the other hand, if $2\left(1-2 d_{1}\right)>1-2 d_{2}$ holds, then the long memory in conditional variance dominates, in the sense that the covariance $\operatorname{Cov}\left(X_{k}^{2}, X_{0}^{2}\right)$ decays as $k^{2 d_{2}-1}$ and $\sum_{t=1}^{N}\left(X_{t}^{2}-E X_{t}^{2}\right)$ converges after normalization to a Gaussian distribution, similarly as in the LARCH model. An econometric discussion of double long memory and other models can be found in Teyssière (2000).

\subsection{EGARCH and Stochastic Volatility Models}

By stochastic volatility ( $S V$ ) one usually means a model of the form

$$
r_{t}=\varepsilon_{t} \sigma_{t}, \quad \varepsilon_{t} \quad \text { i.i.d., } \quad E \varepsilon_{t}=0, \quad \operatorname{Var} \varepsilon_{t}=1,
$$

where $\sigma_{t}>0$ is a measurable function of the past "information set" $F_{t-1}$ which contains all information $r_{s}, \varepsilon_{s} \leq t-1$ up to time $t-1$, and may contain some other "unobservable information" as well, with the property, that $\varepsilon_{s}, s \geq t$ are independent of $F_{t-1}$. This implies of course $E\left(r_{t} \mid F_{t-1}\right)=0$ and $\sigma_{t}^{2}=\operatorname{Var}\left(r_{t} \mid F_{t-1}\right)$. It is often assumed that the volatility is of the form

$$
\sigma_{t}=f\left(\eta_{t}\right)
$$

where $f$ is a (nonlinear) function, and $\eta_{t}$ is a stationary process of some familiar type, e.g. Gaussian or ARMA. 
The choice of $\sigma_{t}$ as a function of Gaussian process imposes distributional assumptions on the volatility which one would like to avoid, of course. On the other hand, it has several important advantages, as explained in Robinson (2001). Firstly, it allows modelling of long memory volatility, by taking $\eta_{t}$ a long memory Gaussian process. Secondly, it allows a very general form of nonlinearity $f$ in (40) by using the techniques of expansions of nonlinear functions of Gaussian random variables in Hermite polynomials. It is clear that a nonlinear function of $\sigma_{t}$, say $\left|\sigma_{t}\right|^{\delta}$, where $\delta>0$ is arbitrary, is again of the form (40) and so this choice of volatility form allows the study of covariance behavior and other properties of general nonlinear transformations of $r_{t}$ and $\sigma_{t}$. An appropriate choice of Gaussian nonlinearity leads to long memory SV models (39) which have infinite fourth moment and for which the autocovariances of $r_{t}^{2}$ are not well-defined. Robinson (2001) discusses a very general class of SV models with Gaussian nonlinearities, where $\varepsilon_{t}=f_{1}\left(\eta_{1 t}\right)$ in (39) is also a nonlinear function of another Gaussian process $\eta_{1 t}$ which is uncorrelated with $\eta_{s}, s \leq t$, and the processes $\eta_{1 t}, \eta_{t}$ may be vector-valued. He obtains the asymptotic formulas for autocovariances of general nonlinear functions of $f_{1}\left(\eta_{1 t}\right) f\left(\eta_{t}\right)$ and exhibits various forms of long memory behavior.

Another popular choice of (40) is $f\left(\eta_{t}\right)=e^{\eta_{t}}$, where $\eta_{t}$ is a moving average process of ARMA or FARIMA type. The corresponding SV model known as the Exponential Generalized $A R C H$ (EGARCH) model, was proposed by Nelson (1991). More precisely, the EGARCH model is given by equations

$$
r_{t}=\sigma_{t} \varepsilon_{t}, \sigma_{t}=\exp \left\{a+\sum_{j=1}^{\infty} b_{j} g\left(\varepsilon_{t-j}\right)\right\}
$$

where

$$
g(z)=\theta z+\gamma\left(|z|-E\left|\varepsilon_{0}\right|\right)
$$

and where $\theta, \gamma$ are parameters which account for certain asymmetries observed in financial data. The particular case $\operatorname{EGARCH}(p, q)$ corresponds to $\eta_{t}=\log \sigma_{t}$ being an $\operatorname{ARMA}(p, q)$ process, i.e. a stationary solution of $\phi(L) \eta_{t}=\psi(L) g\left(\varepsilon_{t}\right)$, where $\phi(z), \psi(z)$ are polynomials of order $p$ and $q$, respectively. The case of $\operatorname{FARIMA}(p, d, q) \eta_{t}$ solving $\phi(L)(1-L)^{d} \eta_{t}=\psi(L) g\left(\varepsilon_{t}\right)$ corresponds to the Fractional Integrated Exponential GARCH (FIEGARCH) model of Bollerslev and Mikkelsen (1996).

A related class of long memory SV models was introduced in Breidt et al. (1998) and Harvey (1998):

$$
r_{t}=\sigma_{t} \varepsilon_{t}, \quad \sigma_{t}=e^{\eta_{t}}, \quad \eta_{t}=a+\sum_{j=1}^{\infty} b_{j} \xi_{t-j},
$$

where $\xi_{t}, t \in \mathbf{Z}$ is a sequence of standard i.i.d. random variables, independent of the sequence $\varepsilon_{t}, t \in \mathbf{Z}$, and where $b_{j}$ are as in FARIMA case.

Long memory properties of powers $\left|r_{t}\right|^{\delta}, \delta>0$ of the SV model of (43) were first obtained in Harvey (1998). In the case when $\xi_{t}$ are normal i.i.d., he 
obtained the autocorrelation function

$$
\operatorname{Cov}\left(\left|r_{t}\right|^{\delta},\left|r_{0}\right|^{\delta}\right)=\left(E\left|r_{0}\right|^{\delta}\right)^{2}\left(e^{\delta^{2} \operatorname{Cov}\left(\eta_{t}, \eta_{0}\right)}-1\right) \sim\left(\delta E\left|r_{0}\right|^{\delta}\right)^{2} \operatorname{Cov}\left(\eta_{t}, \eta_{0}\right),
$$

which shows that the decay of autocovariances of $\left|r_{t}\right|^{\delta}$ is proportional to the decay of autocovariances of (FARIMA) process $\eta_{t}$.

Surgailis and Viano (2002) obtained similar results without imposing distributional assumptions. They considered a generalization of (43), where $\varepsilon_{t}$ and $\xi_{t}$ are not necessary independent; it is only assumed that the bivariate sequence $\left(\varepsilon_{t}, \xi_{t}\right), t \in \mathbf{Z}$ is i.i.d., with zero means $E \varepsilon_{t}=E \xi_{t}=0$ and unit variances. Their model also includes the FIEGARCH model as the particular case $\xi_{t}=g\left(\varepsilon_{t}\right)$. By assuming

$$
b_{j} \sim c_{0} j^{d-1}, \quad\left(c_{0} \neq 0,0<d<1 / 2\right)
$$

and appropriate moment conditions on $\varepsilon_{0}, \xi_{0}$, they proved for any real $\delta>0$

$$
\operatorname{Cov}\left(\left|r_{t}\right|^{\delta},\left|r_{0}\right|^{\delta}\right) \sim\left(\delta E\left|r_{0}\right|^{\delta} c_{1}\right)^{2} t^{2 d-1}
$$

similarly as in (44), where $c_{1}$ depends only on $c_{0}, d$. They also proved the convergence to a fractional Brownian motion:

$$
\left\{N^{-d-1 / 2} \sum_{s=1}^{[N \tau]}\left(\left|r_{s}\right|^{\delta}-E\left|r_{s}\right|^{\delta}\right)\right\} \rightarrow D[0,1] \quad\left\{c_{2} E\left|r_{0}\right|^{u} W_{d}(\tau)\right\}
$$

where $c_{2}=c_{1} /(d(2 d+1))$.

The last paper also discussed the case where the process $\eta_{t}$ in (43) is short memory, in the sense that the coefficients $b_{j}$ are summable: $\sum_{j=1}^{\infty}\left|b_{j}\right|<\infty$. Under similar moment conditions, it proved that in such case the autocovariances of powers $\left|r_{t}\right|^{\delta}$ are also summable, that partial sums of $\left|r_{t}\right|^{\delta}$ converge to a standard Brownian motion, under normalization growing as $N^{1 / 2}$. The proof of the last result uses cumulants and some combinatorial formulas for cumulants of exponents of linear combinations in independent random variables which seem to be new even in the Gaussian case.

As mentioned above, the specific form $\xi_{s}=g\left(\zeta_{s}\right)$ of (42) allows to model certain asymmetries observed in financial data (leverage effect). Nelson (1991) obtained the formula for the covariance between $\log \sigma_{t}$ and $\zeta_{t-k}$. This leverage effect was also discussed by Bollerslev and Mikkelsen (1996). Surgailis and Viano (2002) showed that if the distribution of $\varepsilon_{0}$ in the EGARCH model (41) is symmetric, then the covariance $\operatorname{Cov}\left(\sigma_{t}^{\delta}, r_{t-k}\right)$ has always the sign of the product $\theta b_{k}$, in particular $\operatorname{Cov}\left(\sigma_{t}^{\delta}, r_{t-k}\right)<0$ if $\theta b_{k}<0$.

\subsection{Regime Switching SV and Related Models}

Mikosch and Stărică $(1999,2004)$ argue that the observed long memory in financial data is spurious and can be explained by structural breaks in GARCH 
models. Dahlhaus and Subba Rao (2003) investigated properties and estimation of nonstationary ARCH models with time-varying coefficients. A popular approach to modelling of structural breaks is Markov switching (Hamilton, 1989). Markov switching ARCH models are discussed in Hamilton and Susmel (1994), Cai (1994), Dueker (1997), Francq et al. (2001).

An alternative to Markov regime switching (which generally leads to short memory processes) is renewal regime switching, with independent and heavy tailed consecutive regime durations. Empirical evidence of heavy tailed regime durations is discussed in Jensen and Liu (2001) (lengths of the US business cycle's), Chow and Liu (1999) (dividend series from the CRSP data), Liu (2000) (daily S\&P composite price index). Jensen and Liu (2001), Gourieroux and Jasiak (2001) argue that regime switching with heavy tails may lead to a new forecasting methodology, as an alternative to FARIMA forecasting. Various regime switching models leading to the long memory property and related econometrical issues were discussed in Parke (1999), Granger and Hyung (1999), Diebold and Inoue (2001), Liu (2000), Jensen and Liu (2001), Gourieroux and Jasiak (2001), Leipus and Viano (2003).

Liu (2000), Leipus et al. (2005) discussed regime switching SV model $r_{t}=$ $\sigma_{t} \varepsilon_{t}$, where volatility

$$
\sigma_{t}=\zeta_{j}, \quad S_{j-1}<t \leq S_{j}
$$

assumes i.i.d. values $\zeta_{j}>0$ on random intervals $\left(S_{j-1}, S_{j}\right]$ of a stationary renewal process with i.i.d. durations $U_{j}=S_{j}-S_{j-1}$. If $E \zeta^{2}<\infty$ and the tail distribution $P(U>u)$ decays as

$$
P(U>u) \sim c_{1} u^{-\beta} \quad(u \rightarrow \infty)
$$

with some $c_{1}>0, \beta>1$, then the autocovariance of $\sigma_{t}$ decays as $t^{1-\beta}$ so that for $1<\beta<2$, the SV model in (48) has covariance long memory (Liu, 2000). Moreover, for any $\delta>0$

$$
\left|r_{t}\right|^{\delta}-E\left|r_{t}\right|^{\delta}=\sigma_{t}^{\delta} \nu_{t}+\left(\sigma_{t}^{\delta}-E \sigma_{t}^{\delta}\right) E|\varepsilon|^{\delta},
$$

where $\nu_{t}=\left|\varepsilon_{t}\right|^{\delta}-E\left|\varepsilon_{t}\right|^{\delta}$ are zero mean i.i.d., and $\sigma_{t}^{\delta}$ has the same form (48) with $\zeta_{j}$ replaced by $\zeta_{j}^{\delta}$. Let $E|\varepsilon|^{2 \delta}<\infty, E \zeta^{2 \delta}<\infty$. Then $\sum_{t=1}^{N} \sigma_{t}^{\delta} \nu_{t}=$ $O_{p}\left(N^{1 / 2}\right)$ and the limit distribution of the partial sums process $\sum_{t=1}^{[N \tau]}\left(\left|r_{t}\right|^{\delta}-\right.$ $\left.E\left|r_{t}\right|^{\delta}\right)$ is determined by the second term on the r.h.s. of (50) which is asymptotically $\beta$-stable; more precisely,

$$
\left\{N^{-1 / \beta} \sum_{s=1}^{[N \tau]}\left(\left|r_{s}\right|^{\delta}-E\left|r_{s}\right|^{\delta}\right)\right\} \rightarrow_{\text {f.d.d. }}\left\{Z_{\beta}(\tau)\right\}
$$

in the sense of convergence of finite dimensional distributions, where $Z_{\beta}(\tau)$ is a $\beta$-stable Lévy process with independent increments, see Taqqu and Levy (1986), Pipiras et al. (2004). 
The result (51) is typical for "renewal type long memory" and is in deep contrast with the fBM asymptotics of the corresponding partial sums processes in (47) and (33) for the EGARCH and LARCH models. The limit process $Z_{\beta}(\tau)$ has infinite variance while $\left|r_{t}\right|^{\delta}$ has finite variance, which means an increase of variability in the distributional limit (51). On the other hand, the limit process in (51) has independent increments while the summands have (covarance) long memory, meaning that this long memory does not persist in the distributional limit. A similar lack of persistence of long memory seems characteristic to some other econometric models, in particular to Parke's (1999) model (see Davidson and Sibbertsen, 2002). Similar properties were proved in Mikosch et al. (2002) for some models arising in telecommunications.

A general renewal regime switching scheme leading to a similar "increase of variability" and stable limit distribution of partial sums is discussed in Leipus et al. (2005), in particular, the linear model

$$
X_{t}=\mu_{t}+a_{t} X_{t-1}+\sigma_{t} \varepsilon_{t}
$$

with renewal switching in levels $\left(\mu_{t}\right)$, slope $\left(a_{t}\right)$ and/or volatility $\left(\sigma_{t}\right)$. The case of $\mathrm{AR}(1)$ equation with $a_{t}$ perfoming a heavy tailed regime switching in the interval $[0,1]$ including the unit root $a_{t}=1$ and its neighborhood is discussed in Leipus and Surgailis (2003). Let us note that the existence of covariance long memory in AR(1) model with $a_{t}$ switching between two values 0 and 1 was first observed by Pourahmadi (1988).

As noted above, similar results can be expected for finite memory ARCH models with heavy tailed switching coefficients. The simplest case is the $\operatorname{GARCH}(1,1)$ equation

$$
r_{t}^{2}=\varepsilon_{t}^{2}\left(\alpha_{0 t}+\alpha r_{t-1}^{2}+\beta \sigma_{t-1}^{2}\right),
$$

where $\alpha, \beta$ are nonrandom, and $\left\{\alpha_{0 t}\right\}$ is a (stationary) process, independent of $\left\{\varepsilon_{t}\right\}$. In particular, $\alpha_{0 t}$ may assume only two values 0 and 1 on consecutive intervals of a stationary renewal process with a heavy-tailed inter-renewal distribution $U$, similarly as in Pourahmadi (1988). Equation (52) can be rewritten as

$$
r_{t}^{2}=\alpha_{0 t} r_{0 t}^{2},
$$

where $\left\{r_{0 t}^{2}\right\}$ is a $\operatorname{GARCH}(1,1)$ process with (nonrandom) coefficients $\alpha_{0}=1$, $\alpha, \beta$, which is independent of $\left\{\alpha_{0 t}\right\}$. Clearly,

$$
\operatorname{Cov}\left(r_{t}^{2}, r_{0}^{2}\right)=\left(E r_{00}^{2}\right)^{2} \operatorname{Cov}\left(\alpha_{0 t}, \alpha_{00}\right)+E\left[\alpha_{0 t} \alpha_{00}\right] \operatorname{Cov}\left(r_{0 t}^{2}, r_{00}^{2}\right),
$$

see equation (53) where the second term on the r.h.s. vanishes exponentially, but the first term may decay very slowly, e.g. as $t^{1-\beta}$ in the case of renewal switching $\alpha_{0 t}$ with inter-renewal distribution (49). Moreover, in the above example (52) one can show a similar covariance decay for arbitrary powers $\left|r_{t}\right|^{\delta}$, provided $a_{t}$ assumes values 0,1 only. While this example might be too 
simple and not characteristic, it also demonstrates the possibility of modeling long memory with the help of classical GARCH models with time-varying random coefficients.

\section{Random Coefficient ARCH and Aggregation}

A natural generalization of $\operatorname{GARCH}(1,1)$ (more generally, of $\mathrm{ARCH}(\infty)$ ) is to assume the coefficients $\alpha_{0}, \alpha, \beta$ random and/or time-varying. There exists a considerable literature on random coefficient AR models $(\mathrm{AR}(1)$ in particular), and the interest in such generalizations recently has increased in connection with the studies of models which involve regime switching and structural breaks. The corresponding ARCH models with constant random coefficients lead to non-ergodic processes whose parameters cannot be consistently estimated. The motivation for such studies follows the important Granger's idea of aggregation.

\subsection{Aggregation}

The basic scheme of contemporaneous aggregation usually starts with $N$ "elementary" individual processes $\left\{X_{t}^{(1)}\right\}, \ldots,\left\{X_{t}^{(N)}\right\}$, which evolve according to a random parametric short memory dynamics, and the aggregated process is the limit of the normalized averages $N^{-1} \sum_{i=1}^{N} X_{t}^{(i)}$, as $N \rightarrow \infty$. Granger (1980) found that aggregation of random coefficient autoregressive AR(1) process can lead to Gaussian long memory aggregated process. Since Granger's pioneering work, this question has attracted considerable attention in the econometric and time series literature, see Gonçalves and Gouriéroux (1988), Lippi and Zaffaroni (1999), and Oppenheim and Viano (1999) among others.

A related problem of generating long memory by aggregation of short memory models in ARCH set-up was analyzed in Ding and Granger (1996). They studied the so-called $N$-component $\operatorname{GARCH}(1,1)$ aggregation scheme

$$
r_{N, t}=\sigma_{t} \varepsilon_{t}, \quad \sigma_{t}^{2}=N^{-1} \sum_{i=1}^{N} \sigma_{i, t}^{2}
$$

based on averaging of $\operatorname{GARCH}(1,1)$ volatilities

$$
\sigma_{i, t}^{2}=\sigma^{2}\left(1-\alpha_{i}\right)\left(1-\beta_{i}\right)+\alpha_{i}\left(1-\beta_{i}\right) r_{N, t-1}^{2}+\beta_{i} \sigma_{i, t-1}^{2},
$$

where $\varepsilon_{t}$ are i.i.d. $(0,1)$ random variables and $\alpha_{i}, \beta_{i}$ are i.i.d. random coefficients. Then $\sigma_{i, t}^{2}$ and $\sigma_{t}^{2}$ can be written in $\operatorname{ARCH}(\infty)$ form

$$
\sigma_{i, t}^{2}=a_{0}^{(i)}+\sum_{k=1}^{\infty} a_{k}^{(i)} r_{N, t-k}^{2}, \quad \sigma_{t}^{2}=\bar{a}_{0}+\sum_{k=1}^{\infty} \bar{a}_{k} r_{N, t-k}^{2}
$$


where $a_{0}^{(i)}=\sigma^{2}\left(1-\alpha_{i}\right), a_{k}^{(i)}=\alpha_{i}\left(1-\beta_{i}\right) \beta_{i}^{k-1}$, and $\bar{a}_{k}=N^{-1} \sum_{i=1}^{N} a_{k}^{(i)}$, $k \geq 0$. By ergodicity, $\bar{a}_{k} \rightarrow E a_{k}^{(i)}$. Ding and Granger (1996) conjectured that, similarly as in AR(1) case, the limiting aggregated model

$$
r_{t}^{2}=\varepsilon_{t}^{2} \sigma_{t}^{2}=\varepsilon_{t}^{2}\left(b_{0}+\sum_{k=1}^{\infty} b_{k} r_{t-k}^{2}\right)
$$

which is an $\operatorname{ARCH}(\infty)$ model with deterministic coefficients $b_{k}=E a_{k}^{(i)}$, can exhibit long memory, if $\alpha_{i}$ and $\beta_{i}$ are properly chosen. Further investigation of this model was conducted by Kazakevičius et al. (2004), who assumed that $\sigma^{2}>0$ is a constant, $\alpha_{k}$ are i.i.d. random variables on $[0,1]$ with the mean $E \alpha_{k}=\mu>0$ and $\beta_{k}$ are i.i.d. $\operatorname{Beta}(p, q)$ random variables independent of $\alpha_{k}$. For $p+q=1$ and $0<q<1 / 2$, they showed that

$$
\bar{a}_{0} \rightarrow E a_{0}^{(i)}=\sigma^{2}(1-\mu), \quad \bar{a}_{k} \rightarrow E a_{k}^{(i)}=\mu \tilde{b}_{k}, \quad k \geq 1, \quad \text { a.s. },
$$

where $\tilde{b}_{k}=E\left(1-\beta_{i}\right) \beta_{i}^{k-1} \sim c k^{-q-1}(k \rightarrow \infty)$ are coefficients of the expansion $\sum_{k=1}^{\infty} \tilde{b}_{k} z^{k}=1-(1-z)^{q}, \sum_{k=1}^{\infty} \tilde{b}_{k}=1$. If $\mu \sqrt{\lambda_{2}}<1$, then ${ }^{4} r_{N, t}^{2} \stackrel{L_{2}}{\longrightarrow} r_{t}^{2}=$ $\sigma_{t}^{2} \varepsilon_{t}^{2}$, see Kazakevičius et al. (2004), where the limit $r_{t}^{2}$ is $\operatorname{ARCH}(\infty)$ model with

$$
\sigma_{t}^{2}=\sigma^{2}(1-\mu)+\mu\left(1-(1-L)^{q}\right) r_{t}^{2}=\sigma^{2}(1-\mu)+\mu \sum_{k=1}^{\infty} \tilde{b}_{k} r_{t-k}^{2}
$$

Since the covariance function $r_{t}^{2}$ is absolutely summable, differently from $\mathrm{AR}(1)$ case the aggregation procedure does not lead to the long memory. On the other hand, a similar aggregation procedure of random coefficient GLARCH $(1,1)$ is likely to result in a long memory limiting process, but this possibility was not yet studied.

Leipus and Viano (2002) also discussed the aggregation (averaging) procedure

$$
r_{N, t}^{2}=N^{-1} \sum_{i=1}^{N}\left(r_{t}^{(i)}\right)^{2},
$$

of squared random coefficient $\mathrm{ARCH}$ models

$$
r_{t}^{(i)}=\sigma_{t}^{(i)} \varepsilon_{t},\left(\sigma_{t}^{(i)}\right)^{2}=b_{0}^{(i)}+\sum_{k=1}^{\infty} b_{k}^{(i)}\left(r_{t-k}^{(i)}\right)^{2},
$$

where $\varepsilon_{t}$ are an i.i.d. noise variables and the weights $\left(b_{0}^{(i)}, b_{1}^{(i)}, b_{2}^{(i)} \ldots\right)$ are independent copies of the random sequence $\left(b_{0}, b_{1}, b_{2} \ldots\right)$. They have shown that under additional restrictions on random weights $b_{0}, b_{1}, \ldots$ ensuring existence

\footnotetext{
${ }^{4}$ We say that $\xi_{N} \stackrel{L_{2}}{\longrightarrow} \xi$ if $E\left|\xi_{N}-\xi\right|^{2} \rightarrow 0$ as $N \rightarrow \infty$.
} 
of stationary solution with a finite moment $E r_{t}^{4}<\infty$, the aggregated model can be written as Volterra series

$$
r_{N, t}^{2}=\varepsilon_{t}^{2}\left(\beta_{N}(0)+\sum_{k=1}^{\infty} \sum_{j_{1}, \ldots, j_{k}=1}^{\infty} \beta_{N}\left(j_{1}, \ldots, j_{k}\right) \varepsilon_{t-j_{1}}^{2} \ldots \varepsilon_{t-j_{1}-\cdots-j_{k}}^{2}\right),
$$

with random coefficients $\beta_{N}\left(j_{1}, \ldots, j_{k}\right)=N^{-1} \sum_{i=1}^{N} b_{0}^{(i)} b_{j_{1}}^{(i)} \cdots b_{j_{k}}^{(i)}$. Since $\beta_{N}\left(j_{1}, \ldots, j_{k}\right) \rightarrow \beta\left(j_{1}, \ldots, j_{k}\right)=E\left[b_{0} b_{j_{1}} \cdots b_{j_{k}}\right]$ a.s. by the Strong Law of Large Numbers, for all $j_{1}, \ldots, j_{k} \geq 1$, the aggregated model, as $N \rightarrow \infty$, converges in $L_{2}$ norm to the ARCH model with deterministic coefficients,

$$
r_{N, t}^{2} \stackrel{L_{2}}{\longrightarrow} \bar{r}_{t}^{2}=\varepsilon_{t}^{2}\left(\beta(0)+\sum_{k=1}^{\infty} \sum_{j_{1}, \ldots, j_{k}=1}^{\infty} \beta\left(j_{1}, \ldots, j_{k}\right) \varepsilon_{t-j_{1}}^{2} \ldots \varepsilon_{t-j_{1}-\cdots-j_{k}}^{2}\right)
$$

in view of general result by Kazakevičius et al. (2004) on stability of random coefficient ARCH models. The covariance function of the limit process $\bar{r}_{t}^{2}$ is absolutely summable, i.e. $\bar{r}_{t}^{2}$ has short memory, but the aggregated process $r_{N, t}^{2}$ and the limit process $\bar{r}_{t}^{2}$ are no longer $\mathrm{ARCH}(\infty)$ processes.

Zaffaroni (2000) investigated contemporaneous aggregation of GARCH processes. A number of papers in the ARCH literature deal with the temporal aggregation, partially motivated by the need of bridging between low and high frequency ARCH models (including their continuous time counterparts), see Drost and Nijman (1993), Drost and Werker (1996), Corradi (2000) among others.

Kazakevičius et al. (2004) studied general properties of $\mathrm{ARCH}(\infty)$ random coefficient model $r_{t}$ defined by equations $(2)$, in the case when the coefficients $\left\{b_{0}, b_{1}, \ldots\right\}=: b$ form a sequence of non-negative random variables, and $\varepsilon_{t}, t \in \mathbf{Z}$ is and i.i.d zero mean sequence independent of $b$. Under similar conditions as in the non-random coefficient case, they showed the existence of a stationary solution $r_{t}$ with a finite moment $E r_{t}^{4}$. However, differently from the deterministic coefficient case, in this case the covariance function

$$
\operatorname{Cov}\left(r_{h}^{2}, r_{0}^{2}\right) \rightarrow \operatorname{Var} E^{b} r_{0}^{2}>0
$$

tends as $h \rightarrow \infty$ to a nonzero constant $\operatorname{Var} E^{b} r_{0}^{2}$, where $E^{b}$ denotes a conditional expectation given $b$, which can be easily seen from the equality $\operatorname{Cov}\left(r_{k+h}^{2}, r_{k}^{2}\right)=\operatorname{ECov}^{b}\left(r_{k+h}^{2}, r_{k}^{2}\right)+\operatorname{Var} E^{b} r_{k}^{2}$. Then

$$
E\left(r_{h}^{2}-E^{b} r_{h}^{2}\right)\left(r_{0}^{2}-E^{b} r_{0}^{2}\right) \equiv \operatorname{Cov}^{b}\left(r_{h}^{2}, r_{0}^{2}\right) \rightarrow 0
$$

which implies the convergence $N^{-1} \sum_{h=1}^{N} r_{h}^{2} \stackrel{d}{\longrightarrow} E^{b} r_{0}^{2} \neq E r_{0}^{2}$ to the random limit $E^{b} r_{0}^{2}$, thus showing that the $\mathrm{ARCH}(\infty)$ process $\left\{r_{t}^{2}\right\}$ with constant random coefficients is non-ergodic. 


\section{Statistical Inference}

\subsection{Estimation of Parameters}

Earlier works on (quasi) maximum-likelihood estimation in $\operatorname{GARCH}(p, q)$ model used the assumption of Gaussianity of errors, which was later dropped. Asymptotic properties of such estimators were considered in several papers.

Engle (1982) and Bollerslev (1986) considered the maximum likelihood estimator (MLE) for conditionally $\operatorname{Gaussian} \operatorname{ARCH}(p)$ and $\operatorname{GARCH}(p, q)$ models, which extends readily to $\operatorname{ARCH}(\infty)$ of $(2)$. Given observations $r_{t}$, $t=1, \ldots, N$, the log-likelihood, apart from an additive constant, is equal to

$$
\ell_{N}(\theta, b)=-\frac{1}{2} \sum_{t=1}^{N}\left\{\log \sigma_{t}^{* 2}(\theta, b)+\frac{r_{t}^{2}}{\sigma_{t}^{* 2}(\theta, b)}\right\},
$$

where

$$
\sigma_{t}^{* 2}(\theta, b)=b+\sum_{j=1}^{t-1} b_{j}(\theta) r_{t-j}^{2},
$$

$b$ is any admissible value of $b_{0}$ and $b_{j}(\theta)$ depend on a parameter $\theta \in \mathbf{R}^{p}$. The likelihood (54) was used by Robinson (1991) for testing for long memory in $\operatorname{ARCH}(\infty)$. Note that $(54)$ is only approximate because $\sigma_{t}^{* 2}(\theta, b)$ is not equal to $E_{\theta, b}\left(r_{t}^{2} \mid r_{t-1}, \ldots, r_{1}\right)$.

The MLE of $\left(\theta_{0}, b_{0}\right)$ is defined by

$$
(\widetilde{\theta}, \widetilde{b})=\arg \max \ell_{N}(\theta, b)
$$

where the maximization is taken over a suitable subset of $\mathbf{R}^{p+1}$. For inference, the limiting distribution of $(\widetilde{\theta}, \widetilde{b})$ is of interest. Weiss (1986) showed that $(\widetilde{\theta}, \widetilde{b})$ is $\sqrt{N}$-consistent and asymptotically normal in the case of $\operatorname{ARCH}(p)$ and finite $p$, while Lee and Hansen (1994), Lumsdaine (1986) established similar properties of the MLE in the case of $\operatorname{GARCH}(1,1)$. The asymptotic theory developed by these authors makes significantly weaker assumptions than conditional Gaussianity, by considering $\ell_{N}(\theta, b)$ as a quasi-loglikelihood. The analysis of $\ell_{N}(\theta, b)$ becomes more complicated in the case of $\operatorname{GARCH}(p, q)$ and general $p, q$. Asymptotic properties of the corresponding estimators for general $\operatorname{GARCH}(p, q)$ models were established by Hall and Yao (2003), who used in (54) the following approximation of the conditional variance $\sigma_{t}^{2}=\operatorname{Var}\left(r_{t} \mid r_{1}, \ldots, r_{t-1}\right)$ :

$$
\begin{aligned}
\tilde{\sigma}_{t}(\theta)^{2}= & \frac{\alpha_{0}}{1-\sum_{i=1}^{p} \beta_{i}} \\
& +\sum_{i=1}^{\min (q, t-1)} \alpha_{i}\left(r_{t-i}^{2}+\sum_{k=1}^{t-i-1} \sum_{\substack{1 \leq j_{1}, \ldots, j_{k} \leq p: \\
j_{1}+\ldots+j_{k} \leq t-i}} \beta_{j_{1} \ldots} \beta_{j_{k}} r_{t-i-j_{1}-\ldots-j_{k}}^{2}\right) .
\end{aligned}
$$


Hall and Yao (2003) showed that if $\varepsilon_{t}$ in (65) have finite fourth moment, then the quasi-maximum likelihood estimate of parameters of $\operatorname{GARCH}(p, q)$ is $\sqrt{N}$-consistent and the asymptotic distribution is normal. In the case of heavy-tailed errors with $E \varepsilon_{0}^{4}<\infty$, the class of possible limit distributions is extremely large, the rate of convergence is slower than $N^{1 / 2}$ and the limit distributions of estimators are no longer Gaussian. Hall and Yao (2003) also devised a bootstrap procedure for estimation of parameters of the limit distribution.

Peng and Yao (2003) suggested the modified least absolute deviation estimator

$$
\hat{\theta}_{2}=\arg \min _{\theta} \sum_{t=1}^{n}\left|\log \left(r_{t}^{2}\right)-\log \left(\tilde{\sigma}_{t}^{2}(\theta)\right)\right|
$$

which is less sensitive to heavy-tailed errors. This estimator has the standard $\sqrt{N}$-rate of convergence and the asymptotically normal limit distribution regardless whether the errors are heavy-tailed or not. Their study suggests using of the least absolute deviation estimator $\hat{\theta}_{2}$ when the errors have very heavy tails as $E\left(\left|\varepsilon_{t}\right|^{3}\right)<\infty$, and using the quasi-maximum likelihood estimator $\tilde{\theta}$ as long as $\varepsilon_{t}$ are not very heavy tailed. Peng and Yao (2003) also investigated asymptotic properties of the absolute deviation estimator $\hat{\theta}_{1}=\arg \min _{\theta} \sum_{t=1}^{n}\left|r_{t}^{2} / \tilde{\sigma}_{t}^{2}(\theta)-1\right|$, and by the regression motivated estimator $\hat{\theta}_{3}=\arg \min _{\theta} \sum_{t=1}^{n}\left|r_{t}^{2}-\tilde{\sigma}_{t}^{2}(\theta)\right|$. Let us note that asymptotic normality of quasi-maximum likelihood estimator of parameters of $\operatorname{GARCH}(p, q)$ under mild moment conditions was obtained in Berkes et al. (2003a), Berkes and Horváth (2004).

Straumann and Mikosch (2003) discussed quasi-maximum likelihood estimation in a general heteroscedastic time series model $X_{t}=\sigma_{t} \varepsilon_{t}$, where unobservable volatility $\sigma_{t}$ is a parametric function of $\left(X_{t-1}, \sigma_{t-1}\right)$, and $\varepsilon_{t}$ is a standard i.i.d. noise. They showed the existence and uniqueness of a stationary solution $X_{t}$, established the consistency and asymptotic normality of the estimator and applied their results to $\operatorname{GARCH}(1,1)$, asymmetric $\operatorname{GARCH}(1,1)$ and EGARCH models. For some recent results, see also Berkes et al. (2003a), Francq and Zakoïan (2004), Ling and McAleer (2003b).

Giraitis and Robinson (2001) considered Gaussian or Whittle estimation based on $r_{t}^{2}, t=1, \ldots, N$. The idea of such estimation in the GARCH case was pointed out by Bollerslev (1986) who noted that $r_{t}^{2}$ generated by (65) has an ARMA type representation, albeit with conditionally heteroscedastic innovations, and it was employed in Harvey (1998) and Robinson and Zaffaroni $(1997,1998)$ for certain class of stochastic volatility and non-linear moving average processes.

Rewrite $\mathrm{ARCH}(\infty)$ model (2) as

$$
r_{t}^{2}=b_{0}+\sum_{j=1}^{\infty} b_{j}(\theta) r_{t-j}^{2}+\nu_{t},
$$


where $\nu_{t}=r_{t}^{2}-\sigma_{t}^{2}$ are martingale differences $b_{j}(\theta)$ are depend on a parameter $\theta$. Assuming $r_{t}$ is a fourth-order stationary sequence, under some additional conditions $r_{t}^{2}$ has spectral density

$$
f(\lambda)=\frac{\sigma^{2}}{2 \pi} g(\lambda, \theta), \quad g(\lambda, \theta)=\left|1-\sum_{j=1}^{\infty} b_{j}(\theta) e^{i j \lambda}\right|^{-2}, \quad-\pi<\lambda \leq \pi,
$$

where $\sigma^{2}:=E \nu_{t}^{2}$. Consider the objective function

$$
w_{N}(\theta)=\sum_{j=1}^{N-1} \frac{I\left(\lambda_{j}\right)}{g\left(\lambda_{j} ; \theta\right)}
$$

where $I(\lambda)=\frac{1}{2 \pi N}\left|\sum_{t=1}^{N} r_{t}^{2} e^{i t \lambda}\right|^{2}$ is the periodogram of the $r_{t}^{2}, \lambda_{j}=2 \pi j / N$. The Whittle estimate is defined by

$$
\widehat{\theta}=\arg \min _{\theta \in \Theta} w_{N}(\theta),
$$

where $\Theta$ is a compact subset of $\mathbf{R}^{p}$. The normalized spectral density $g(\lambda ; \theta)$ is often explicitly given, for example, for $\operatorname{GARCH}(p, q)$ models it equals (see Bollerslev, 1986)

$$
g(\lambda ; \theta)=\left|\frac{1-\beta\left(e^{i \lambda}\right)}{1-\alpha\left(e^{i \lambda}\right)-\beta\left(e^{i \lambda}\right)}\right|^{2},
$$

where $\alpha(z)=\sum_{j=1}^{q} \alpha_{j} z^{j}, \beta(z)=\sum_{j=1}^{p} \beta_{j} z^{j}$. Under some additional regularity and moment conditions and the normalizing assumption

$$
\int_{-\pi}^{\pi} \log g(\lambda ; \theta) d \lambda=0,
$$

Giraitis and Robinson (2001) showed that

$$
N^{1 / 2}\left(\widehat{\theta}-\theta_{0}\right) \stackrel{d}{\longrightarrow} N\left(0,2 W^{-1}+W^{-1} V W^{-1}\right)
$$

where

$$
W=\frac{1}{2 \pi} \int_{-\pi}^{\pi} \frac{\partial \log g\left(\lambda ; \theta_{0}\right)}{\partial \theta} \frac{\partial \log g\left(\lambda ; \theta_{0}\right)}{\partial \theta^{\prime}} d \lambda
$$

and

$$
V=\frac{2 \pi}{\sigma^{2}} \int_{-\pi}^{\pi} \int_{-\pi}^{\pi} \frac{\partial g\left(\lambda ; \theta_{0}\right)^{-1}}{\partial \theta} \frac{\partial g\left(\omega ; \theta_{0}\right)^{-1}}{\partial \theta^{\prime}} f(\lambda,-\omega, \omega) d \lambda d \omega,
$$

where $f(\lambda, \omega, \nu)$ is the fourth-order cumulant spectrum of $r_{t}^{2}$. Note that the matrices $V$ and $W$ can be consistently estimated.

The following two remarks should be made in this context. Firstly, the above Whittle estimation in the case of $\operatorname{GARCH}(p, q)$ processes requires that their spectral density (56) is sufficiently regular and satisfies condition (57). 
Secondly, the above results apply to weights which decrease much more slowly than exponentially, e.g. as

$$
b_{j}=c_{1} j^{-\zeta},
$$

where $\zeta>1$ and $c_{1}>0$, in which case we have in the above example, $\theta=$ $\left(\theta_{1}, \theta_{2}\right)$ and

$$
g(\lambda ; \theta)=\left|1-\left(\theta_{1}-1\right) \theta_{2} \sum_{j=1}^{\infty} j^{-\theta_{1}} e^{i j \lambda}\right|^{-2},
$$

where the true $\theta_{1}$ equals $\zeta, \theta_{2}$ is bounded by 1 from above, and $g(\lambda ; \theta)$ satisfies (57). Of course, the lack of a closed form representation of the above spectral density is a practical disadvantage.

Although the Whittle estimation method is simple and easy to use, $\widehat{\theta}$ has a different limiting variance from $\widetilde{\theta}$ as shown in Lee and Hansen (1994), Lumsdaine (1996), and is asymptotically less efficient than $\widetilde{\theta}$ when the $r_{t}$ 's are conditionally Gaussian.

Let us note, finally, the result of Mikosch and Straumann (2002), who showed some non-standard properties of the Whittle estimator in a heavytailed $\operatorname{GARCH}(1,1)$ model.

\subsection{Change-point Problem}

In order to avoid spurious inference, an important problem in financial data analysis is testing for parameter constancy against some kind of instability in the conditional variance, in particular for structural breaks of variance $\operatorname{Var} r_{t}$.

Kokoszka and Leipus (1999) studied the CUSUM type tests for a changepoint in the parameters of the conditional variance of the $\mathrm{ARCH}(\infty)$ model defined by equations (2), which results in the change of the variance $\operatorname{Var} r_{t}$. They assumed the following condition

$$
\left(E \varepsilon_{0}^{8}\right)^{1 / 4} \sum_{j=1}^{\infty} b_{j}<1
$$

guarantying the existence of an eighth order strictly stationary solution $r_{t}$ and the validity of the Functional Limit Theorem for the squares $r_{t}^{2}$.

Denote by $b:=\left(b_{0}, b_{1}, \ldots\right)$ the parameter sequence of $\operatorname{ARCH}(\infty)$ process $r_{t}^{2},(2)$, and write $\left\{r_{t}\right\} \in \mathcal{R}(b)$ if $b$ satisfies condition (58).

Suppose we want to test the null hypothesis

$$
H_{0}: r_{1}^{2}, \ldots, r_{N}^{2} \text { is a sample from }\left\{r_{t}\right\} \in \mathcal{R}(b) \text { for some } b
$$

against the alternative that the sample $r_{1}^{2}, \ldots, r_{N}^{2}$ has the form

$$
r_{t}^{2}=\left\{\begin{array}{lll}
\left(r_{t}^{(1)}\right)^{2}, & \text { if } & 1 \leq t \leq k^{*} \\
\left(r_{t}^{(2)}\right)^{2}, & \text { if } & k^{*}<t \leq N
\end{array}\right.
$$


where $k^{*}=\left[N \tau^{*}\right], 0<\tau^{*}<1$ is fixed, and sequences $\left\{r_{t}^{(1)}\right\} \in \mathcal{R}\left(b^{(1)}\right)$, $\left\{r_{t}^{(2)}\right\} \in \mathcal{R}\left(b^{(2)}\right)$ with $b^{(1)} \neq b^{(2)}$ have different variances $\operatorname{Var} r_{t}^{(1)} \neq \operatorname{Var} r_{t}^{(2)}$. The sequences $\left\{r_{t}^{(1)}\right\}$ and $\left\{r_{t}^{(2)}\right\}$ are generated by the same noise sequence $\left\{\varepsilon_{t}\right\}$.

Kokoszka and Leipus (1999) studied CUSUM type tests based on the process $\left\{U_{N}(\tau), \tau \in[0,1]\right\}$, where

$$
U_{N}(\tau)=N^{1 / 2} \frac{[N \tau](N-[N \tau])}{N^{2}}\left(\frac{1}{[N \tau]} \sum_{j=1}^{[N \tau]} r_{j}^{2}-\frac{1}{N-[N \tau]} \sum_{j=[N \tau]+1}^{N} r_{j}^{2}\right)
$$

The partial sums in (60) are empirical estimates of the variance of the $r_{t}$, so the tests are designed to detect a change in parameters which leads to a change in variance of returns.

Under the null hypothesis $H_{0}$,

$$
\left\{U_{N}(\tau), \tau \in[0,1]\right\} \rightarrow D[0,1]\left\{\sigma W^{0}(\tau), \tau \in[0,1]\right\}
$$

where $\sigma^{2}=\sum_{k=-\infty}^{\infty} \operatorname{Cov}\left(r_{k}^{2}, r_{0}^{2}\right)$ and $W^{0}(\tau)=W(\tau)-\tau W(1)$ is a Brownian bridge. Under $H_{0}$, this implies the convergence of the following standard statistics

$$
\sup _{0 \leq \tau \leq 1}\left|U_{N}(\tau)\right| \stackrel{d}{\longrightarrow} \sigma \sup _{0 \leq \tau \leq 1}\left|W^{0}(\tau)\right|, \quad \int_{0}^{1} U_{N}^{2}(\tau) d \tau \stackrel{d}{\longrightarrow} \sigma^{2} \int_{0}^{1}\left(W^{0}(\tau)\right)^{2} d \tau .
$$

The tests based on such continuous functionals of the process $\left\{U_{N}(\tau)\right\}$ have positive asymptotic power against the alternative $H_{1}$.

Note that under $H_{0}$, the process $r_{t}^{2}$ has short memory and $\sigma^{2}<\infty$. To construct the asymptotic critical regions, the only unknown parameter $\sigma^{2}$ of the limit distributions in (61) can be estimated by

$$
\hat{s}_{N, q}^{2}=\sum_{|j| \leq q}\left(1-\frac{|j|}{q+1}\right) \hat{\gamma}(j)
$$

where $q \rightarrow \infty, q / N \rightarrow 0$ is the bandwidth parameter, $\hat{\gamma}(j)=N^{-1} \sum_{i=1}^{N-|j|}\left(r_{i}^{2}-\right.$ $\left.\bar{r}^{2}\right)\left(r_{i+|j|}^{2}-\bar{r}^{2}\right)$ are the sample covariances and $\bar{r}^{2}=N^{-1} \sum_{j=1}^{N} r_{j}^{2}$ is the sample mean. Then, as $N \rightarrow \infty$,

$$
\hat{s}_{N, q}^{2} \stackrel{P}{\longrightarrow} \sigma^{2} .
$$

To estimate the change-point $\tau^{*}$ Kokoszka and Leipus (2000) have used the estimator $\hat{\tau}=\hat{k} / N$, which is based on the CUSUM statistic (60), setting

$$
\hat{k}=\min \left\{k:\left|U_{k}\right|=\max _{1 \leq j<N}\left|U_{j}\right|\right\},
$$

where $U_{k}=U_{N}(k / N)$. Then 


$$
\left|\hat{\tau}-\tau^{*}\right|=O_{P}\left(\frac{1}{N \Delta^{4}}\right),
$$

where $\Delta=E\left(r_{k}^{(2)}\right)^{2}-E\left(r_{k}^{(1)}\right)^{2} \neq 0$. Here $E\left(r_{k}^{(i)}\right)^{2}=\lambda_{1} b_{0}^{(i)} /\left(1-\lambda_{1} \sum_{j=1}^{\infty} b_{j}^{(i)}\right)$, $i=1,2$.

Andreou and Ghysels $(2002,2003)$ applied these results to the stock and FX markets. Tests for changes in volatility, based on squared model residuals and applicable to the GARCH model were designed by Kokoszka and Teyssière (2002); for related research see Horvàth et al. (2001), Berkes and Horváth (2003a), Berkes et al. (2003b). Berkes et al. (2004) used a test based on the likelihood ratio. Sequential monitoring scheme for detection of changes in the parameters of GARCH sequence model was studied by Berkes, Gombay, Horváth and Kokoszka (2004).

\section{References}

1. Andersen, T. G., Bollerslev, T., Diebold, F. X. and Labys, P. (2001) The distribution of realized exchange rate volatility. Journal of American Statistical Association, 96, 42-55.

2. Andreou, E. and Ghysels, E. (2002). Detecting multiple breaks in financial market volatility dynamics. Journal of Empirical Econometrics, 17, 579-600.

3. Andreou, E. and Ghysels, E. (2003). Tests for breaks in the conditional comovements of asset returns. Statistica Sinica, 13, 1045-1073.

4. Baillie, R. T., Bollerslev, T. and Mikkelsen, H. O. (1996a). Fractionally integrated generalized autoregressive conditional heteroskedasticity. Journal of Econometrics, 74, 3-30.

5. Baillie, R. T., Chung, C.-F. and Tieslau, M. A. (1996b). Analysing inflation by the fractionally integrated ARFIMA-GARCH model. Journal of Applied Econometrics, 11, 23-40.

6. Barlow, R. E. and Proschan, F. (1981). Statistical Theory of Reliability and Life: Probability Models. Silver Spring, MD.

7. Bera, A. and Higgins, M. L. (1993). ARCH models: properties, estimation and testing. Journal of Economic Surveys, 7, 305-366.

8. Berkes, I., Gombay, E., Horvàth, L. and Kokoszka, P. S. (2004). Sequential change-point detection in $\operatorname{GARCH}(p, q)$ models. Econometric Theory, 20, 11401167

9. Berkes, I., Horvàth, L. and Kokoszka, P. S. (2002b). Probabilistic and statistical properties of GARCH processes. Fields Institute Communications, forthcoming.

10. Berkes, I. and Horvàth, L. (2003a). Limit results for the empirical process of squared residuals in GARCH models. Stochastic Processes and their Applications, 105, 271-298.

11. Berkes, I. and Horvàth, L. (2003b). Asymptotic results for long memory LARCH sequences. Annals of Applied Probability, 13, 641-668.

12. Berkes, I. and Horvàth, L. (2004). The efficiency of the estimators of the parameters in GARCH processes. Annals of Statistics, 32, 633-655.

13. Berkes, I., Horvàth, L. and Kokoszka, P. S. (2003a). GARCH processes: structure and estimation. Bernoulli, 9, 201-227. 
14. Berkes, I., Horvàth, L. and Kokoszka, P. S. (2003b). Asymptotics for GARCH squared residual correlations. Econometric Theory, 19, 515-540.

15. Berkes, I., Horvàth, L. and Kokoszka, P. S. (2004). Testing for parameter constancy in $\operatorname{GARCH}(p, q)$ models. Statistics and Probability Letters, 70, 263-273,

16. Black, F. (1976). Studies in stock price volatility changes. Proceedings of the 1976 Business Meeting of the Business and Economic Statistics Section. American Statistical Association, 177-181.

17. Bollerslev, T. (1986). Generalized autoregressive conditional heteroskedasticity. Journal of Econometrics, 31, 307-327.

18. Bollerslev, T. (1988). On the correlation structure for the generalized autoregressive conditional heteroskedastic process. Journal of Time Series Analysis, 9, 121-131.

19. Bollerslev, T., Chou, R.Y. and Kroner, K.F. (1992). ARCH modeling in finance: a review of the theory and empirical evidence. Journal of Econometrics, 52, 559.

20. Bollerslev, T., Engle, R. F. and Nelson, D. B. (1994). ARCH models. In: Handbook of Econometrics, New York (eds R. F. Engle and D. L. McFadden), volume 4, pp. 2961-3031. Elsevier Science.

21. Bollerslev, T. and Mikkelsen, H. O. (1996). Modeling and pricing long memory in stock market volatility. Journal of Econometrics, 73, 151-184.

22. Bougerol, P. and Picard, N. (1992). Stationarity of GARCH processes and of some nonnegative time series. Journal of Econometrics, 52, 115-127.

23. Breidt, F. J., Crato, N. and de Lima, P. (1998). On the detection and estimation of long memory in stochastic volatility. Journal of Econometrics, 83, 325-348.

24. Cai, J. (1994). A Markov model of switching-regime ARCH. Journal of Business E Economic Statistics, 12, 309-316.

25. Chow, Y.-F. and Liu, M. (1999). Long swings with memory and stock market fluctuations. Journal Financial and Quantitative Analysis, 34, 341-367.

26. Corradi, V. (2000). Reconsidering the continuous time limit of the $\operatorname{GARCH}(1,1)$ process. Journal of Econometrics, 96, 145-153.

27. Cox, J. T. and Grimmett, G. (1984). Central limit theorems for associated random variables and the percolation models. Annals of Probability, 12, 514528.

28. Dacorogna, M. M., Müller, U. A., Nagler, R. J., Olsen, R. B. and Pictet, O. V. (1993). A geographical model for the daily and weekly seasonal volatility in the foreign exchange market. Journal of International Money and Finance, 12, 413-438.

29. Dahlhaus, R. and Subba Rao, S. (2003). Statistical inference for time-varying ARCH processes. Annals of Statistics, forthcoming.

30. Davidson, J. (2004). Moment and memory properties of linear conditional heteroscedasticity models, and a new model. Preprint.

31. Davidson, J. and Sibbertsen, Ph. (2002). Generating schemes for long memory processes: regimes, aggregation and linearity. Preprint.

32. Diebold, F.X. and Inoue, A. (2001). Long memory and regime switching. Journal of Econometrics, 105, 131-159.

33. Ding, Z. and Granger, C. W. J. (1996). Modeling volatility persistence of speculative returns: a new approach. Journal of Econometrics, 73, 185-215.

34. Ding, Z., Granger, C. W. J. and Engle, R. F. (1993). A long memory property of stock market returns and a new model. Journal of Empirical Finance, 1, 83-106. 
35. Drost, F. C. and Nijman, T. E. (1993). Temporal aggregation of GARCH processes. Econometrica, 61, 909-927.

36. Drost, F. C. and Werker, B. (1996). Closing the GARCH gap: continuous time GARCH modeling. Journal of Econometrics, 74, 31-57.

37. Dueker, M. J. (1997). Markov switching in GARCH processes and meanreverting stock-market volatility. Journal of Business $\mathcal{E}$ Economic Statistics, 15, 26-34.

38. Engle, R. F. (1982). Autoregressive conditional heteroscedasticity with estimates of the variance of United Kingdom inflation. Econometrica, 50, 987-1008.

39. Engle, R. F. (1990). Stock volatility and the crash of '87. discussion. The Review of Financial Studies, 3, 103-106.

40. Engle, R. F. and Bollerslev, T. (1986). Modelling the persistence of conditional variances. Econometric Reviews, 27, 1-50.

41. Engle, R. F. and Russell, J. R. (1998). Autoregressive conditional duration: a new model for irregularly spaced transaction data. Econometrica, 66, 11271162.

42. Fan, J. and Yao, Q. (2002). Non-linear Time Series: Nonparametric and Parametric Methods. Springer, New York.

43. Francq, C. and Zakoïan, J.-M. (2004). Maximum likelihood estimation of pure GARCH and ARMA-GARCH processes. Bernoulli, 10, 605-637,

44. Francq, C., Roussignol, M. and Zakoïan, J.-M. (2001). Conditional heteroskedasticity driven by hidden Markov chains. Journal of Time Series Analysis, 22, 197-220.

45. Giraitis, L., Kokoszka, P. and Leipus, R. (2000a). Stationary ARCH models: dependence structure and Central Limit Theorem. Econometric Theory, 16, $3-22$.

46. Giraitis, L., Kokoszka, P., Leipus, R. and Teyssière, G. (2000b). Semiparametric estimation of the intensity of long memory in conditional heteroskedasticity. Statistical Inference for Stochastic Processes, 3, 113-128.

47. Giraitis, L., Robinson, P. M. and Surgailis, D. (2000c). A model for long memory conditional heteroskedasticity. Annals of Applied Probability, 10, 1002-1024.

48. Giraitis, L., Kokoszka, P., Leipus, R. and Teyssière, G. (2003). Rescaled variance an related tests for long memory in volatility and levels. Journal of Econometrics, 112, 265-294.

49. Giraitis, L., Leipus, R., Robinson, P. M. and Surgailis, D. (2004). LARCH, leverage and long memory. Journal of Financial Econometrics, 2, 177-210.

50. Giraitis, L. and Robinson, P. M. (2001). Whittle estimation of ARCH models. Econometric Theory, 17, 608-631.

51. Giraitis, L. and Surgailis, D. (2002). ARCH-type bilinear models with double long memory. Stochastic Processes and their Applications, 100, 275-300.

52. Gonçalves, E. and Gouriéroux, C. (1988). Aggrégation de processus autoregressifs d'ordre 1. Annales d'Economie et de Statistique, 12, 127-149.

53. Gouriéroux, C. ARCH Models and Financial Applications. Springer, Berlin.

54. Gourieroux, C. and Jasiak, J. (2001). Memory and infrequent breaks. Economics Letters, 70, 29-41.

55. Granger, C.W.J. (1980). Long memory relationships and the aggregation of dynamic models. Journal of Econometrics, 14, 227-238.

56. Granger, C. W. J. and Andersen, A. P. (1978). An Introduction to Bilinear Time Series Models. Vandenhoek \& Ruprecht, Göttingen. 
57. Granger, C. W. J. and Hyung, N. (1999). Occasional structural breaks and long memory. Discussion paper 99-14, Department of Economics. University of California, San Diego.

58. Hall, P. and Yao, Q. (2003). Inference in ARCH and GARCH models with heavy-tailed errors. Econometrica, 71, 285-317.

59. Hamilton, J. D. and Susmel, R. (1994). Autoregressive conditional heteroskedasticity and changes in regime. Journal of Econometrics, 64, 307-333.

60. Harvey, A. (1998). Long memory in stochastic volatility. In: Forecasting Volatility in the Financial Markets, Oxford (eds J. Knight and S. Satchell), pp. 307-320. Butterworth \& Heineman.

61. He, C. and Teräsvirta, T. (1999). Fourth moment structure of the $\operatorname{GARCH}(p, q)$ process. Econometric Theory, 15, 824-846.

62. Horváth, L., Kokoszka, P. and Teyssière, G. (2001). Empirical process of the squared residuals of an ARCH sequence. Annals of Statistics, 29, 445-469.

63. Jensen, M. J. and Liu, M. (2001). Do long swings in the business cycle lead to strong persistence in output? Journal of Monetary Economics, forthcoming.

64. Karanasos, M. (1999). The second moment and the autocovariance function of the squared errors of the GARCH model. Journal of Econometrics, 90, 63-76.

65. Kazakevičius, V. and Leipus, R. (2002). On stationarity in the $\mathrm{ARCH}(\infty)$ model. Econometric Theory, 18, 1-16.

66. Kazakevičius, V. and Leipus, R. (2003). A new theorem on existence of invariant distributions with applications to ARCH processes. Journal of Applied Probability, 40, 147-162.

67. Kazakevičius, V., Leipus, R. and Viano, M.-C. (2004). Stability of random coefficient autoregressive conditionally heteroskedastic models and aggregation schemes. Journal of Econometrics, 120, 139-158.

68. Kokoszka, P. and Leipus, R. (1999). Testing for parameter changes in ARCH models. Lietuvos Matematikos Rinkinys, 39, 231-247.

69. Kokoszka, P. and Leipus, R. (2000). Change-point estimation in ARCH models. Bernoulli, 6, 513-539.

70. Kokoszka, P. and Teyssière, G. (2002). Change-point detection in GARCH models: asymptotic and bootstrap tests. Preprint.

71. Koulikov, D. (2003). Modeling sequences of long memory non-negative covariance stationary random variables. Preprint.

72. Lee, S.-W. and Hansen, B. E. (1994). Asymptotic theory for the GARCH $(1,1)$ quasi-maximum likelihood estimator. Econometric Theory, 10, 29-52.

73. Leipus, R., Paulauskas, V. and Surgailis, D. (2005). Renewal regime switching and stable limit laws. Journal of Econometrics, 129, 299-327.

74. Leipus, R. and Surgailis, D. (2003). Random coefficient autoregression, regime switching and long memory. Advances in Applied Probability, 35, 737-754.

75. Leipus, R. and Viano, M.-C. (2002). Aggregation in ARCH models. Lietuvos Matematikos Rinkinys, 42, 68-89.

76. Leipus, R. and Viano, M.-C. (2003). Long memory and stochastic trend. Statistics and Probability Letters, 61, 177-190.

77. Li, W. K., Ling, S. and McAleer, M. (2002). Recent theoretical results for time series models with GARCH errors. Journal of Economic Surveys, 16, 245-269.

78. Ling, S. and Li, W. K. (1998). On fractionally integrated autoregressive moving average time series models with conditional heteroskedasticity. Journal of the American Statistical Association, 92, 1184-1193. 
79. Ling, S. and McAleer, M. (2002a). Stationarity and the existence of moments of a family of GARCH processes. Journal of Econometrics, 106, 109-117.

80. Ling, S. and McAleer, M. (2002b). Necessary and sufficient moment conditions for the $\operatorname{GARCH}(r, s)$ and asymmetric power $\operatorname{GARCH}(r, s)$ models. Econometric Theory, 18, 722-729.

81. Ling, S. and McAleer, M. (2003a). Asymptotic theory for a vector ARMAGARCH model. Econometric Theory, 19, 278-308.

82. Ling, S. and McAleer, M. (2003b). On adaptive estimation in nonstationary ARMA models with GARCH errors. Annals of Statistics, 31, 642-674.

83. Lippi, M. and Zaffaroni, P. (1999). Contemporaneous aggregation of linear dynamic models in large economies. Preprint.

84. Liu, M. (2000). Modeling long memory in stock market volatility. Journal of Econometrics, 99, 139-171.

85. Louichi, S. (1998). Théorèmes limites pour des suites positivement ou faiblement dépendantes. PhD thesis, Université de Paris Sud Orsay, 1998.

86. Lumsdaine, R. L. (1996). Consistency and asymptotic normality of the quasilikelihood estimator in $\operatorname{IGARCH}(1,1)$ and covariance stationary $\operatorname{GARCH}(1,1)$ models. Econometrica, 64, 575-596.

87. Mikosch, T. (2003). Modelling dependence and tails of financial time series. In: Extreme Values in Finance, Telecommunications and the Environment (eds B. Finkenstädt and H. Rootzén), pp. 185-286. Chapman \& Hall.

88. Mikosch, T., Resnick, S., Rootzén, H. and Stegeman, A. (2002). Is network traffic approximated by stable Lévy motion or fractional Brownian motion? Annals of Applied Probability, 12, 23-68.

89. Mikosch, T. and Stărică, C. (1999). Change of structure in financial time series, long range dependence and the GARCH model. Preprint.

90. Mikosch, T. and Stărică, C. (2000). Is it really long memory we see in financial returns? In: Extremes and Integrated Risk Management, London (ed. P. Embrechts), pp. 149-168. Risk Books.

91. Mikosch, T. and Stărică, C. (2003). Long-range dependence effects and ARCH modeling. In Theory and Applications of Long-Range Dependence, Boston (eds P. Doukhan, G. Oppenheim and M. S. Taqqu), pp. 439-459. Birkhäuser.

92. Mikosch, T. and Stărică, C. (2004). Non-stationarities in financial time series, the long-range dependence and the IGARCH effects. Review of Economics and Statistics, 86, 378-390.

93. Mikosch, T. and Straumann, D. (2002). Whittle estimation in a heavy-tailed GARCH(1,1) model. Stochastic Process and their Applications, 100, 187-222.

94. Nelson, D. B. (1990). Stationarity and persistence in the $\operatorname{GARCH}(1,1)$ model. Econometric Theory, 6, 318-334.

95. Nelson, D. B. (1991). Conditional heteroskedasticity in asset returns: a new approach. Econometrica, 59, 347-370.

96. Nelson, D. B. and Cao, C. Q. (1992). Inequality constraints in the univariate GARCH model. Journal of Business 85 Economic Statistics, 10, 229-235.

97. Newman, C. M. (1984). Asymptotic independence and limit theorems for positively and negatively dependent random variables. In Inequalities in Statistics and Probability (ed. Tong, Y. L.), Hayward, CA: Inst. Math. Stat.

98. Newman, C. M. and Wright, A. L. (1981). An invariance principle for certain dependent sequences. Annals of Probability, 9, 671-675. 
99. Oppenheim, G. and Viano. M.-C. (1999). Obtaining long memory by aggregating random coefficients discrete and continuous time simple short memory processes. PUB. IRMA, LILLE, Vol. 49, No. V.

100. Pagan, A. (1996). The econometrics of financial markets. Journal of Empirical Finance, 3, 15-102.

101. Parke, W. R. (1999). What is fractional integration? The Review of Economics and Statistics, 81, 632-638.

102. Peng, L. and Yao, Q. (2003). Least absolute deviations estimation for ARCH and GARCH models. Biometrika, 90, 967-975.

103. Pipiras, V., Taqqu, M. S. and Levy, J.B. (2004). Slow, fast and arbitrary growth conditions for the renewal reward processes when the renewals and the rewards are heavy-tailed. Bernoulli, 10, 378-390.

104. Pourahmadi, M. (1988). Stationarity of the solution of $X_{t}=A_{t} X_{t-1}+\varepsilon_{t}$ and analysis of non-Gaussian dependent variables. Journal of Time Series Analysis, 9, 225-239.

105. Robinson, P. M. (1991). Testing for strong serial correlation and dynamic conditional heteroskedasticity in multiple regression. Journal of Econometrics, 47, $67-84$.

106. Robinson, P. M. (2001). The memory of stochastic volatility models. Journal of Econometrics, 101, 195-218.

107. Robinson, P. M. and Zaffaroni, P. (1997). Modelling nonlinearity and long memory in time series. Fields Institute Communications, 11, 161-170.

108. Robinson, P. M. and Zaffaroni, P. (1998). Nonlinear time series with long memory: a model for stochastic volatility. Journal of Statistical Planning and Inference, 68, 359-371.

109. Rydberg, T. (2000). Realistic statistical modelling of financial data. International Statistical Review, 68, 233-258.

110. Sentana, E. (1995). Quadratic ARCH models. Review of Economic Studies, 62, 639-661.

111. Shephard, N. (1996). Statistical aspects of ARCH and stochastic volatility. In Time Series Models in Econometrics, Finance and Other Fields (eds D. R. Cox, D. V. Hinkley and O. E. Barndorff-Nielsen), pp. 1-55. Chapman \& Hall.

112. Straumann, D. and Mikosch, T. (2003). Quasi-maximum-likelihood estimation in heteroscedastic time series: a stochastic recurrence equations approach. $A n$ nals of Statistics, forthcoming.

113. Subba Rao, T. and Gabr, M. M. (1984). An Introduction to Bispectral Analysis and Bilinear Time Series Models. Lecture Notes in Statistics, volume 24. Springer Verlag, New York.

114. Surgailis, D. and Viano, M.-C. (2002). Long memory properties and covariance structure of the EGARCH model. ESAIM: Probability and Statistics, 6, 311329.

115. Taqqu, M.S. and Levy, J.B. (1986). Using renewal processes to generate longrange dependence and high variability. In Dependence in Probability and Statistics (eds E. Eberlein and M. S. Taqqu), pp. 73-89. Birkhäuser, Boston.

116. Taylor, S. (1986). Modelling Financial Time Series. Wiley, New York.

117. Teräsvirta, T. (1996). Two stylized facts and the GARCH(1,1) model. Stockholm School of Economics. SSE/EFI Working Paper Series in Economics and Finance, No. 96. 
118. Terdik, G. (1999). Bilinear Stochastic Models and Related Problems of Nonlinear Time Series Analysis: A Frequency Domain Approach. Lecture Notes in Statistics, volume 142. Springer Verlag, New York.

119. Teyssière, G. (2000). Double long-memory financial time series. Preprint.

120. Tsay, R. S. (2002). Analysis of Financial Time Series. Wiley, New York.

121. Weiss, A. A. (1986). Asymptotic theory for ARCH models: estimation and testing. Econometric Theory, 2, 107-131.

122. Zaffaroni, P. (2000). Contemporaneous aggregation of GARCH processes. Preprint. 